\title{
COUNTING INTERVAL GRAPHS
}

BY

PHIL HANLON

\begin{abstract}
In this paper we enumerate interval graphs (up to isomorphism) along with labelled interval graphs, identity interval graphs, transitive interval graphs and various sorts of unit interval graphs. The enumeration makes use of a structural decomposition of interval graphs which leads to a characterization of those interval graphs having a unique interval representation. Several tables are included.
\end{abstract}

1. Preliminaries. Let $G=(V(G), E(G))$ be a graph with $n$ points. We say that $G$ is an interval graph if there exists a 1-1 function $\Pi$ mapping $V(G)$ onto a set of $n$ real closed intervals which satisfies the following condition $(*)$.

(*) for all $u, v \in V(G),\{u, v\} \in E(G)$ iff $\Pi(u)$ and $\Pi(v)$ have nonempty intersection.

If (*) holds we call $\Pi$ an interval representation of $G$. The aim of this paper is to count the number of graphs on $n$ points which are interval graphs.

Not every graph is an interval graph; the smallest exception is the 4-cycle. Bohland and Lekkerkerker [2] characterized those graphs which are interval graphs in terms of forbidden subgraphs. A second characterization, also in terms of forbidden subgraphs, was obtained by Gilmore and Hoffman [5]. For a summary of work done on the subject of interval graphs, see Golumbic [6].

Let $\Pi$ be an interval representation of the graph $G$. It is clear that the intervals $\Pi(u)$ for $u \in V(G)$ can be chosen so that no two share a common endpoint. We will deal exclusively with such representations. We use the notation $l(\Pi u)$ and $r(\Pi u)$ to denote the left-hand and right-hand endpoints of the interval $\Pi u$. The notation $\Pi u<\Pi v$ means $r(\Pi u)<l(\Pi v)$.

We henceforth assume that the vertex set of $G$ consists of the integers from 1 to $n$. Let $\Pi$ be a representation of $G$; from $\Pi$ we construct a linear ordering of the set of pairs $\{(i, s): 1 \leqslant i \leqslant n, s=0,1\}$. This ordering, called the characteristic ordering of $\Pi$, is given by the rules

(a) $(i, 0)<(j, 0)$ iff $l(\Pi i)<l(\Pi j)$;

(b) $(i, 0)<(j, 1)$ iff $l(\Pi i)<r(\Pi j)$;

(c) $(i, 1)<(j, 1)$ iff $r(\Pi i)<r(\Pi j)$.

Two representations are considered the same if they have the same characteristic ordering. Hence there are only a finite number of different representations of each interval graph.

Received by the editors October 28, 1980.

1980 Mathematics Subject Classification. Primary 05A15. 
For example, let $G$ be the interval graph below:

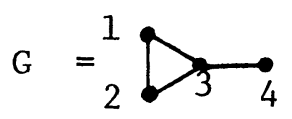

Let $\Pi$ be the following representation of $G$ :

$$
\begin{array}{ll}
\Pi(1)=[1,5], & \Pi(2)=[2,4], \\
\Pi(3)=[3,7], & \Pi(4)=[6,8] .
\end{array}
$$

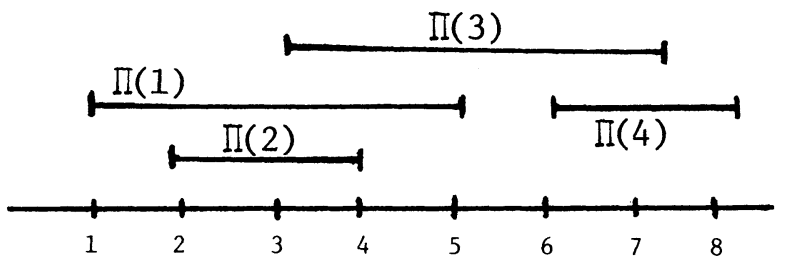

The characteristic ordering of this representation is

$$
(1,0)<(2,0)<(3,0)<(2,1)<(1,1)<(4,0)<(3,1)<(4,1) .
$$

A sequence of consecutive left-hand endpoints in the characteristic ordering of $\Pi$ is a sequence $\left(i_{1}, 0\right)<\left(i_{2}, 0\right)<\cdots<\left(i_{k}, 0\right)$ having the property that no pair of the form $(j, 1)$ lies between any $\left(i_{l}, 0\right)$ and $\left(i_{l+1}, 0\right)$. Visually this means that no right-hand endpoint lies in the interval $\left[l \Pi i_{1}, l \Pi i_{k}\right]$. A sequence of consecutive right-hand endpoints is defined analogously.

If the characteristic ordering of $\rho$ can be obtained from the characteristic ordering of $\Pi$ by rearranging sequences of consecutive right-hand and left-hand endpoints then we say $\Pi$ and $\rho$ are strongly similar. It is clear that strong similarity is an equivalence relation and that if $\Pi$ and $\rho$ are strongly similar then they represent the same graph.

Let $\Pi$ be a representation of $G$. The opposite of $\Pi$, denoted $\Pi^{\prime}$, is the unique representation of $G$ which satisfies the following 4 conditions:

(a) $l\left(\Pi^{\prime} u\right)<l\left(\Pi^{\prime} v\right)$ iff $r(\Pi u)>r(\Pi v)$;

(b) $l\left(\Pi^{\prime} u\right)<r\left(\Pi^{\prime} v\right)$ iff $r(\Pi u)>l(\Pi v)$;

(c) $r\left(\Pi^{\prime} u\right)<l\left(\Pi^{\prime} v\right)$ iff $l(\Pi u)>r(\Pi v)$;

(d) $r\left(\Pi^{\prime} u\right)<r\left(\Pi^{\prime} v\right)$ iff $l(\Pi u)>l(\Pi v)$.

For example, if

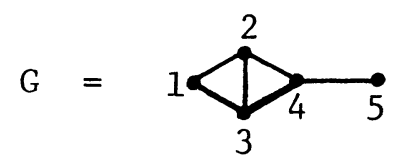


and $\Pi$ is

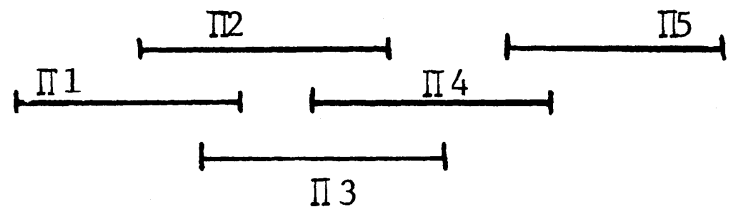

then $\Pi^{\prime}$ is

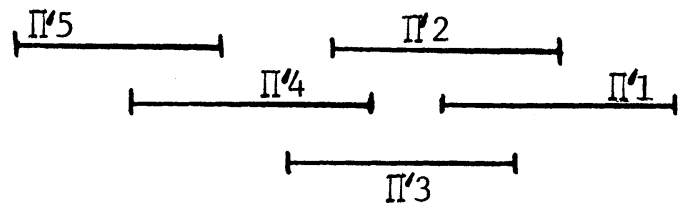

One should think of obtaining $\Pi^{\prime}$ from $\Pi$ by flipping the real line across a point which lies in the middle of the representation $\Pi$. If $\Pi$ and $\Pi^{\prime}$ are strongly similar then $\Pi$ is called symmetric and we say that $\Pi$ is invariant under a flip of $\mathbf{R}$.

Two representations $\Pi$ and $\rho$ are similar if either $\Pi$ and $\rho$ are strongly similar or $\Pi$ and $\rho^{\prime}$ are strongly similar. Similar representations obviously yield the same graph; unfortunately dissimilar representations may yield the same graph as well. The key to our enumeration will be to characterize those interval graphs which have only one representation up to similarity.

If $G$ is a graph and $u$ and $v$ are adjacent points then we say that $u$ and $v$ have the same neighbors provided that for all $w \in V(G)-\{u, v\}$ we have $\{u, w\} \in E(G)$ if $\{v, w\} \in E(G)$. Other graph theory terminology follows Harary [9].

2. Dependable interval graphs. In this section we give a structural characterization of those interval graphs which have only one interval representation up to similarity.

Definition 1. Let $G$ be a graph and let $S$ be a subset of $V(G)$. By $K(S)$ we mean the (perhaps empty) set of points of $G$ which are adjacent to every point in $S$; i.e.

$$
K(S)=\{v \in V(G):\{v, s\} \in E(G) \text { for all } s \in S\} .
$$

For an arbitrary graph $G$, little can be said about these sets $K(S)$. However, if $G$ is an interval graph, then more can be said.

Proposition 1. Let $G$ be an interval graph and let $S \subseteq V(G)$. If $S$ contains two nonadjacent points then either $K(S)$ is empty or the subgraph of $G$ induced by $K(S)$ is complete.

Proof. Choose an interval representation $\Pi$ of $G$ and choose nonadjacent points $u$ and $v$ in $S$. Suppose $K(S)$ is nonempty; we will show that every pair of points in $K(S)$ are adjacent in $G$.

As $u$ and $v$ are nonadjacent either $\Pi u<\Pi v$ or $\Pi v<\Pi u$; assume the former. If $w_{1}$ and $w_{2}$ are points in $K(S)$ then $\Pi u \cap \Pi w_{1}$ is nonempty and $\Pi v \cap \Pi w_{1}$ is nonempty so the right-hand endpoint $r(\Pi u)$ is in the interval $\Pi w_{1}$. Similarly $r(\Pi u) \in \Pi w_{2}$ and so $\Pi w_{1} \cap \Pi w_{2}$ is nonempty. Thus, $w_{1}$ and $w_{2}$ are adjacent in $G$. 
Proposition 1 will frequently be used without mention. As in the proof of Proposition 1, the same letter $S$ will be used to denote a subset of $V(G)$ and the subgraph of $G$ induced by $S$.

DEFINITION 2. Let $G$ be an interval graph and let $B$ be a subset of $V(G)$. We call $B$ a buried subgraph of $G$ if

(a) The set $B$ is nonempty and the subgraph of $G$ induced by $B$ is noncomplete.

(b) $K(B)$ is nonempty and $B \cap K(B)=\varnothing$.

(c) If $u$ is a point in $B, v$ is a point not in $B$ and $u=v_{0}, v_{1}, \ldots, v_{n}=v$ is a $u$-v path in $G$ then $v_{i} \in K(B)$ for some $i$.

Condition (c) states that $K(B)$ separates $B$ from the rest of $G$ in the sense that the only way to enter or leave $B$ is through $K(B)$.

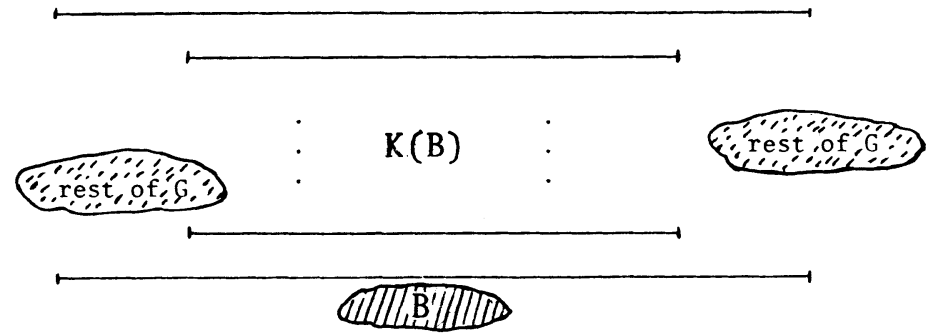

A connected interval graph with no buried subgraphs is called dependable. Later in this section we will show that each dependable interval graph has a unique interval representation. To do so we need two technical lemmas.

LEMMA 1. Let $G$ be a connected interval graph and let $\Pi$ and $\rho$ be representations of $G$. Suppose that for vertices $a, b$ and $c$ we have

$$
\Pi a<\Pi b<\Pi c \text { and } \rho a<\rho c<\rho b .
$$

Then $K(\{b, c\})$ is nonempty, and every path from $a$ to $b$ contains a point of $K(\{b, c\})$.

Proof. Let $a=v_{0}, v_{1}, \ldots, v_{m}=b$ be an $a-b$ path in $G$. One exists since $G$ is connected. Let $i$ be such that $\Pi v_{i} \cap \Pi b \neq \varnothing$ but $\Pi v_{\kappa} \cap \Pi b=\varnothing$ for all $k<i$. As $\Pi a<\Pi b<\Pi c, \Pi v_{\kappa} \cap \Pi c=\varnothing$ for $k<i$. We will show that $\Pi v_{i} \cap \Pi c$ is nonempty.

Suppose that $\Pi v_{i} \cap \Pi c=\varnothing$. Then $a=v_{0}, v_{1}, \ldots, v_{i}, b$ is an $a-b$ path in $G$ containing no points adjacent to $c$. Hence $\rho a=\rho v_{0}, \rho v_{1}, \ldots, \rho v_{i}, \rho b$ is a sequence of overlapping intervals none of which intersect $\rho c$. But this contradicts $\rho a<\rho c<\rho b$ and so $v_{i} \in K(\{b, c\})$ which proves the lemma.

The next lemma contains the essence of what we want to prove. Again we consider two representations, $\Pi$ and $\rho$, of the same dependable interval graph $G$. The lemma deals specifically with those pairs of points $u, v \in V(G)$ which are nonadjacent and hence whose corresponding intervals are ordered both in $\Pi$ and in $\rho$. The content of the lemma is that this ordering of nonadjacent points must either be exactly the same in $\rho$ as in $\Pi$ or exactly opposite. 
LEMMA 2. Let $G$ be a dependable interval graph, and let $\Pi$ and $\rho$ be representations of $G$. Then either

(a) for all points $u, v \in V(G)$ we have $\Pi u<\Pi v$ iff $\rho u<\rho v$ or

(b) for all points $u, v \in V(G)$ we have $\Pi u<\Pi v$ iff $\rho u>\rho v$.

Proof. Suppose not; assume that there exist points $u_{1}, u_{2}, v_{1}$ and $v_{2}$ in $V(G)$ with

$$
\Pi u_{1}<\Pi v_{1}, \quad \rho u_{1}<\rho v_{1} \text { and } \Pi u_{2}<\Pi v_{2}, \quad \rho u_{2}>\rho v_{2} .
$$

Observe that these hypotheses insure that $G$ is not a complete graph.

Choose $l$ and $r$ in $V(G)$ such that $\Pi l$ and $\Pi r$ are the intervals in $\Pi$ with the smallest right-hand and largest left-hand endpoints respectively. As $G$ is not complete, $\Pi l<\Pi r$. Let $S$ be the set of points of $G$ with $\Pi v<\Pi r$ and $\rho v>\rho r$.

Case 1. $S$ is nonempty and $l \notin S$ (so $\rho l<\rho r$ ). Choose $t$ in $S$ so that $\Pi t$ has the smallest right-hand endpoint amongst the intervals $\Pi v$ for $v \in S$.

Let $B^{\prime}=\{b: r(\Pi b) \geqslant r(\Pi t)\}$ and let $B=B^{\prime}-K\left(B^{\prime}\right)$. We will show that $B$ is a buried subgraph of $G$; we verify the necessary conditions in order.

(1) The points $r$ and $t$ are in $B$ and as $t \in S$ we have $\Pi t<\Pi r$. So $B$ is nonempty and induces a noncomplete subgraph of $G$.

(2) Observe first that $\Pi l<\Pi t$. For if $\Pi l \cap \Pi t$ was nonempty then $\rho l \cap \rho t$ would be nonempty which would imply that $\rho r<\rho l$ and this would contradict that $l \notin S$.

Thus $\Pi l<\Pi t<\Pi r$ and $\rho l<\rho r<\rho t$ and so, by Lemma $1, K(\{r, t\})$ is nonempty. By our choice of $r$ and $t$ we have $K(B)=K(\{r, t\})=K\left(B^{\prime}\right)$ so $K(B)$ is nonempty and $B \cap K(B)=B \cap K\left(B^{\prime}\right)=\varnothing$.

(3) Let $u$ be a point in $B$ and let $v$ be a point not in $B$. If $v$ is in $K(B)$ then certainly every $u-v$ path contains a point in $K(B)$, namely $v$ itself. We therefore assume that $v$ is not in $K(B)$, so $v \notin B \cup K(B)=B^{\prime}$ and so $r(\Pi v)<r(\Pi t)$.

By our choice of $t, \rho v<\rho r$; hence $\rho v \cap \rho t=\varnothing$. So $\Pi v \cap \Pi t=\varnothing$ and $\Pi v<\Pi t$. Thus we have

$$
\Pi v<\Pi t<\Pi r, \quad \rho v<\rho r<\rho t .
$$

Now let $v=v_{0}, v_{1}, \ldots, v_{n}=u$ be a $v-u$ path in $G$.

We show that $\Pi v_{m} \cap \Pi t$ is nonempty for some $m \leqslant n$. We need only consider the case where $\Pi u \cap \Pi t=\varnothing$. As $u \in B, r(\Pi t)<r(\Pi u)$ and so $\Pi t<\Pi u$. But now as $\Pi v<\Pi t<\Pi u$, it is clear that $\Pi v_{m} \cap \Pi t \neq \varnothing$ for some $m$.

Thus, we have that $v=v_{0}, v_{1}, \ldots, v_{m}, t$ is a $v$ - $t$ path in $G$ and so using Lemma 1 and the inequalities (2.1) we see that $v_{i} \in K(\{r, t\})=K(B)$ for some $i \leqslant m \leqslant n$.

So $B$ is a buried subgraph of $G$ which contradicts our assumption that $G$ is dependable. Thus Case 1 cannot occur.

Case 2. $l$ is an element of $S$ (so $\rho l>\rho r$ ). Let $R=\{(a, b): \Pi a<\Pi b$ and $\rho a<\rho b\}$. Note that $R$ is nonempty by hypothesis. Choose $c_{0}$ so that $\Pi c_{0}$ has the smallest right-hand endpoint amongst the intervals $\Pi a$ where $a$ appears in the first coordinate of a pair in $R$. Choose $d_{0}$ so that $\Pi d_{0}$ has the largest left-hand endpoint amongst the intervals $\Pi b$ where the pair $\left(c_{0}, b\right)$ is in $R$.

Define the set $B^{\prime}$ to be

$$
B^{\prime}=\left\{v: l(\Pi v) \leqslant l\left(\Pi d_{0}\right) \text { and } r(\Pi v)>r\left(\Pi c_{0}\right)\right\} .
$$

Let $B=B^{\prime}-K\left(B^{\prime}\right)$. 
It is straightforward to check that $B$ is a buried subgraph of $G$ and so Case 2 cannot happen.

Case 3. $S$ is empty.

Let $R=\{(a, b): \Pi a<\Pi b$ and $\rho a>\rho b\}$.

Again $R$ is nonempty by hypothesis. Choose $c_{0}$ so that $\Pi c_{0}$ has the smallest right-hand endpoint amongst the intervals $\Pi a$ where $a$ appears in the first coordinate of a pair in $R$. Choose $d_{0}$ so that $\Pi d_{0}$ has the largest left-hand endpoint amongst the intervals $\Pi b$ where $\left(c_{0}, b\right) \in R$. Define the set $B^{\prime}$ to be

$$
B^{\prime}=\left\{v: l(\Pi v) \leqslant l\left(\Pi d_{0}\right) \text { and } r(\Pi v) \geqslant r\left(\Pi c_{0}\right)\right\},
$$

and let $B=B^{\prime}-K\left(B^{\prime}\right)$.

Again it is not hard to show that $B$ is a buried subgraph of $G$ and so Case 3 cannot happen. As Cases 1-3 exhaust the possibilities, this completes the proof of Lemma 2.

The following theorem constitutes our goal for this section.

THEOREM 1. Let $G$ be a dependable interval graph. Then $G$ has exactly one interval representation (up to similarity).

Proof. Let $\Pi$ and $\rho$ be representations of $G$. According to Lemma 2, we may assume (by reflecting the $\rho$-representation over $|V(G)|+\frac{1}{2}$ if necessary), that $\Pi u<\Pi v$ iff $\rho u<\rho v$. We will show that $\Pi$ and $\rho$ are the same up to a rearrangement of consecutive right- and left-hand endpoints.

To prove this we will define the sets $\Pi U_{1}, \ldots, \Pi U_{n}$ and $\Pi L_{1}, \ldots, \Pi L_{n}$ for $n=|V(G)|$ as follows;

(1) $\Pi U_{1}$ contains the intervals whose right-hand endpoints appear in the smallest run of consecutive right-hand endpoints in $\Pi$.

(2) $\Pi U_{i+1}$ contains the intervals whose right-hand endpoints appear in the smallest run of consecutive right-hand endpoints above the run corresponding to $\Pi U_{i}$.

Define $\Pi L_{i}$ identically except using left-hand endpoints. For example

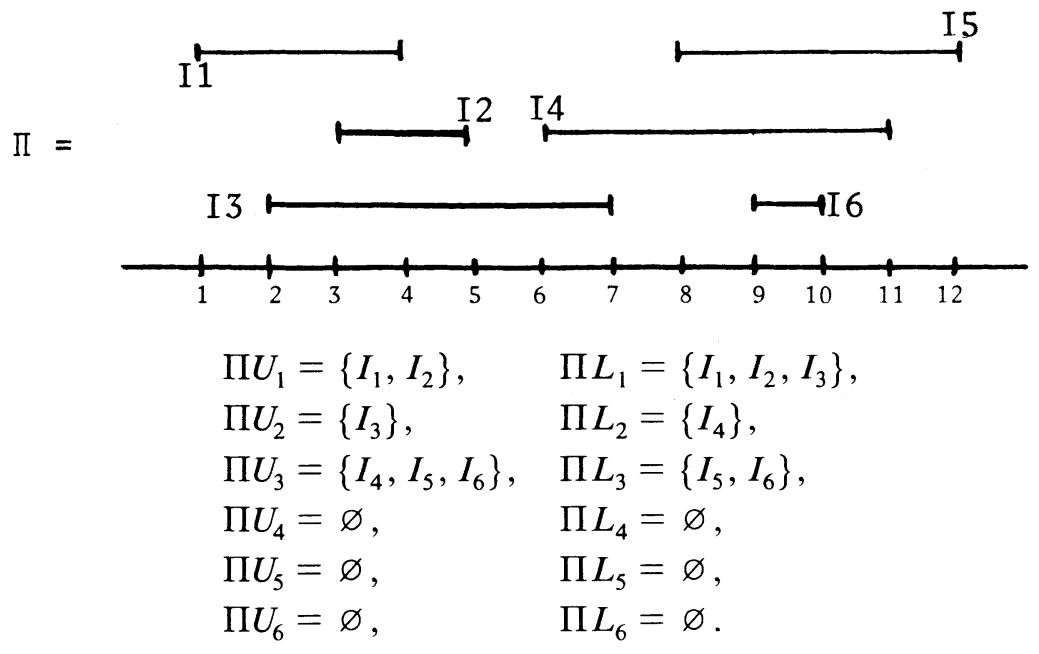


Similarly define the sets $\rho U_{i}$ and $\rho L_{i}$. Clearly it suffices to show that, for all $i$, $\Pi U_{i}=\rho U_{i}$ and $\Pi L_{i}=\rho L_{i}$. But

$$
\begin{aligned}
\Pi L_{1} & =\{v: \Pi w \nless \Pi v \text { for all } w \in V(G)\} \\
& =\{v: \rho w \nless \rho r \text { for all } w \in v(G)\}=\rho L_{1} .
\end{aligned}
$$

Next,

$$
\begin{aligned}
\Pi U_{1} & =\left\{v: \Pi w>\Pi v \text { iff } w \in V(G)-\Pi L_{1}\right\} \\
& =\left\{v: \rho w>\rho v \text { iff } w \in V(G)-\rho L_{1}\right\}=\rho U_{1} .
\end{aligned}
$$

In a similar fashion, $\Pi L_{2}=\rho L_{2}, \Pi U_{2}=\rho U_{2}$ and so on. This completes the proof of Theorem 1.

COROLlaRY 1. Let $G$ be a dependable interval graph.

If $G$ has a symmetric representation then every representation of $G$ is symmetric.

It should be noted that a similar result does not hold in general. For example the graph $G$ below has the two representations $\Pi$ and $\rho$. $\Pi$ is symmetric and $\rho$ is not.

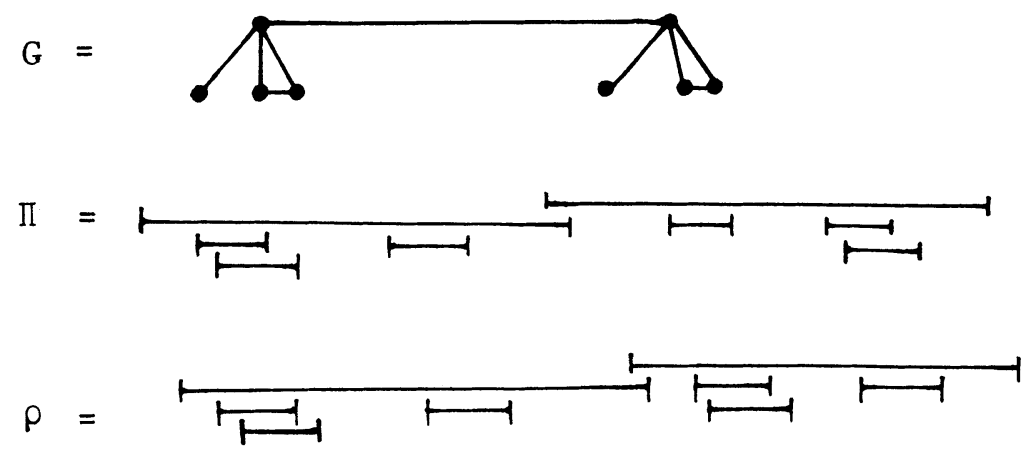

In this section we actually proved a slightly stronger result than is stated in Theorem 1; this stronger version will be used in $\S 6$. In fact we showed that if $\Pi$ and $\rho$ are representations of a dependable interval graph $G$ then either

(A) $\Pi u<\Pi v$ iff $\rho u<\rho v$ in which case $\Pi$ and $\rho$ are strongly similar, or

(B) $\Pi u<\Pi v$ iff $\rho u>\rho v$ in which case $\Pi$ and $\rho^{\prime}$ are strongly similar.

3. A reduction procedure. In this section, we assign each connected interval graph to a unique dependable interval graph. This is done in such a way that it is possible to determine, with a minimum of information, the number of connected interval graphs assigned to each dependable interval graph. We begin with a technical lemma.

LEMMA 3. Let $G$ be a connected interval graph and let $B_{1}$ and $B_{2}$ be buried subgraphs of $G$.

(1) If $B_{1} \cap B_{2}$ is nonempty then $B_{1} \cup B_{2}$ is a buried subgraph of $G$.

(2) If $B_{1} \cap K\left(B_{2}\right)$ is nonempty then $B_{1} \cup B_{2}$ is a buried subgraph of $G$.

Proof. We first prove (1). If either $B_{1} \subseteq B_{2}$ or $B_{2} \subseteq B_{1}$ the result is obvious. Consider the case where neither inclusion holds; let $\Pi$ be a representation of $G$. 
Assume first that $K\left(B_{1}\right)-K\left(B_{2}\right)$ is nonempty. Let $k \in K\left(B_{1}\right)-K\left(B_{2}\right)$ and let $v \in B_{1} \cap B_{2}$. For each $u \in B_{1}-B_{2}, u, k, v$ is a path from outside $B_{2}$ into $B_{2}$ which therefore contains a point in $K\left(B_{2}\right)$. By choice, $k \notin K\left(B_{2}\right)$ and so $u$ must be in $K\left(B_{2}\right)$. Since this is true for any $u \in B_{1}-B_{2}$ we have shown that $B_{1}-B_{2} \subseteq K\left(B_{2}\right)$.

Let $u_{0}$ be a fixed element of $B_{1}-B_{2}$. Then $\Pi u_{0} \cap \Pi u$ is nonempty for all $u \in B_{1}-B_{2}$ since we have just shown that $B_{1}-B_{2}$ is a subgraph of the complete graph $K\left(B_{2}\right)$. On the other hand, $\Pi u_{0} \cap \Pi b$ is nonempty for all $b$ in $B_{1} \cap B_{2}$ and so $u_{0} \in B_{1} \cap K\left(B_{1}\right)$. This is a contradiction; hence $K\left(B_{1}\right)-K\left(B_{2}\right)$ is empty.

Similarly $K\left(B_{2}\right)-K\left(B_{1}\right)$ is empty and so $K\left(B_{1}\right)=K\left(B_{2}\right)=K\left(B_{1} \cup B_{2}\right)$. Using this fact it is a simple matter to check that $B_{1} \cup B_{2}$ is buried.

We now prove (2). If $B_{1} \cap B_{2} \neq \varnothing$ we are done by (1). Assume that $B_{1} \cap B_{2}$ is empty and let $b_{1}$ be an element of the set $B_{1} \cap K\left(B_{2}\right)$. Let $c$ be any element of $B_{2}$. Then $c, b_{1}$ is a path from outside $B_{1}$ into $B_{1}$ which therefore contains a point of $K\left(B_{1}\right)$. As $b_{1} \in B_{1}$ this point cannot be $b_{1}$; hence $c \in K\left(B_{1}\right)$. So $B_{2} \subseteq K\left(B_{1}\right)$ which means $B_{2}$ is complete and this is a contradiction.

Definition 3. A buried subgraph $B$ is maximal if $B \cup S$ fails to be buried for all nonempty sets $S \subseteq V(T)-B$.

THEOREM 2. Let $G$ be an interval graph and let $B_{1}$ and $B_{2}$ be maximal buried subgraphs of $G$. Then either $B_{1}=B_{2}$ or $B_{1} \cap B_{2}=\varnothing$.

Proof. If $B_{1} \cap B_{2}$ is nonempty then $B_{1} \cup B_{2}$ is a buried subgraph of $G$ and so, using that each is maximal, we have $B_{1}=B_{2}$.

It is now clear that we may assign to every connected interval graph a unique dependable interval graph by simply removing each maximal buried subgraph $B$ and replacing $B$ by a single interval.

DeFinition 4. Let $G$ be a connected interval graph and let $S=\left\{B_{1}, B_{2}, \ldots, B_{n}\right\}$ (perhaps empty) be the set of maximal buried subgraphs of $G$. Define an interval graph $\delta(P)$ by

(1) $V(\delta(P))=\left(V(G)-\left(\cup_{i=1}^{n} B_{i}\right)\right) \cup\left\{v_{B_{1}}, \ldots, v_{B_{n}}\right\}$ where $\left\{v_{B_{1}}, \ldots, v_{B_{n}}\right\}$ is a set of $n$ new vertices-one for each maximal buried subgraph of $G$.

(2) $E(\delta(P))$ consists of all those pairs $\{u, v\}$ such that either both $u$ and $v$ are in the set $V(G)-\left(\cup B_{i}\right)$ and $\{u, v\} \in E(G)$, or $u \in V(G)-\left(\cup B_{i}\right), v=v_{B_{l}}$ and $u \in K\left(B_{l}\right)$ for some $l$.

For example,

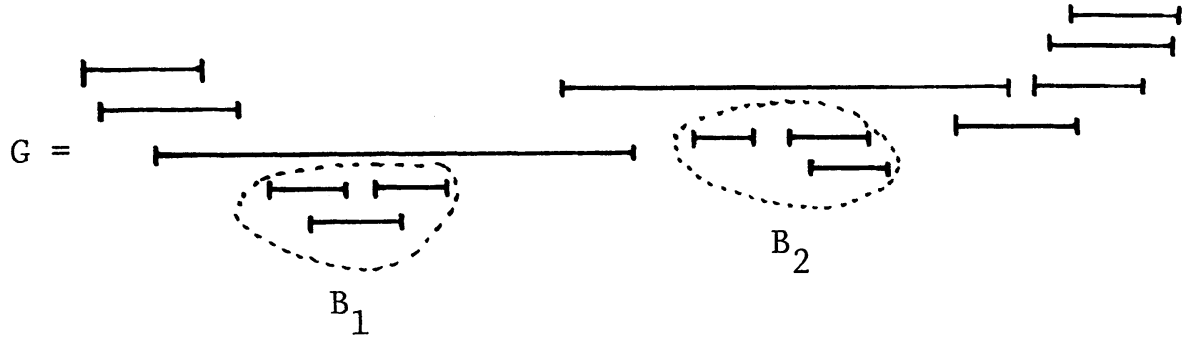


then

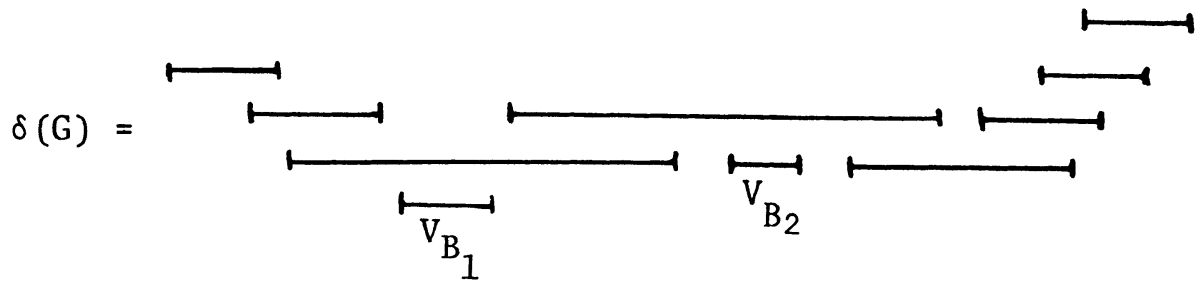

It is easy to check that $\delta(G)$ is connected and that $\delta(G)$ contains no buried subgraphs. The graph $\delta(G)$ will be called the dependable image of $G$.

To ease the upcoming generating function arguments, it is necessary to make one further reduction. This time we squash together stacks of intervals which sit directly on top of each other, i.e. identify all intervals which have exactly the same neighbors.

Definition 5. Let $G$ be a dependable interval graph. We say $G$ is reduced if no two distinct points of $G$ have exactly the same neighbors.

For example, of the two dependable interval graphs below, only $G$ is reduced.

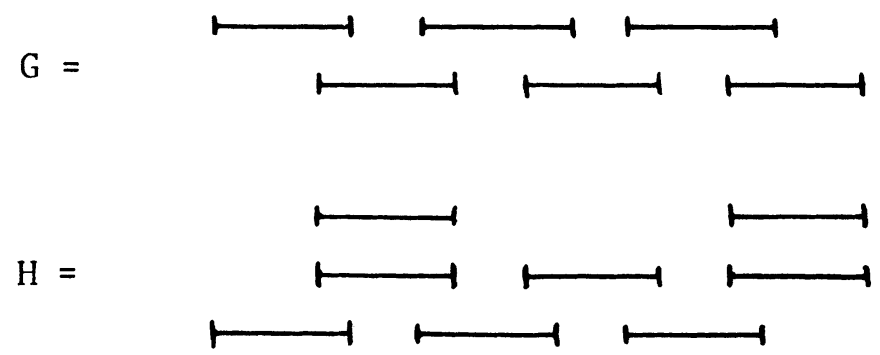

Given a dependable interval graph we obtain a reduced interval graph by identifying intervals which have exactly the same neighbors. When this is done to $\delta(G)$, the dependable image of an interval graph $G$, the resulting graph will be called the reduced image of $G$ and denoted $\nu(G)$.

We now have enough terminology to easily describe which interval graphs have a unique interval representation. Let $\mathfrak{A}$ be the set of connected interval graphs defined inductively as follows:

(1) If $G$ is dependable then $G$ is in $\mathfrak{A}$ iff $G$ has a symmetric representation.

(2) If $G$ is connected but not dependable then $G$ is in $\mathfrak{A}$ iff $G$ has a symmetric representation and every maximal buried subgraph of $G$ is in $\mathfrak{A}$.

Then a connected interval graph $G$ is uniquely representable up to strong similarity iff $G \in \mathfrak{A}$.

Next let $\mathfrak{B}$ be the set of connected interval graphs defined inductively as follows:

(1) Every dependable interval graph is in $\mathfrak{B}$.

(2) If $G$ is not dependable then $G$ is in $\mathscr{B}$ iff every maximal buried subgraph of $G$ is in $\mathfrak{A}$. 
Then an interval graph $G$ is uniquely representable up to similarity iff $G \in \mathfrak{B}$. This answers questions posed by Fred Roberts (see Roberts [15, 16]).

The structure results given in $\$ \S 2$ and 3 imply facts about automorphism groups of interval graphs, which will be used implicitly in the generating function manipulations which follow. To end this section we prove those results that will be of later use.

If $G$ is a reduced interval graph then $G$ has at most two representations up to strong similarity by Theorem 1 . If $G$ has only one representation then any representation $\Pi$ must be strongly similar to its opposite $\Pi^{\prime}$, i.e. $\Pi$ must be symmetric.

Proposition 2. Let $G$ be a reduced interval graph with more than one point. Then either

(a) $G$ has two representations up to strong similarity and automorphism group of size one or

(b) $G$ has only one representation up to strong similarity and automorphism group of size 2. In this case, if $\sigma$ is the nontrivial automorphism of $G$ and if $\Pi$ is any representation of $G$ then $\Pi^{\prime}$ is strongly similar to the representation $\rho$ given by $\rho(u)=\Pi(u \sigma)$.

Proof. Let $G$ be reduced, let $\Pi$ be a representation of $G$ and let $\sigma$ be an automorphism of $G$. Define a new representation $\rho$ of $G$ as follows:

$$
\rho(u)=\Pi(u \sigma) .
$$

Since $\sigma$ is an automorphism of $G, \rho$ is a representation of $G$. By Theorem 1 we know that $\rho$ is strongly similar to either $\Pi$ or $\Pi^{\prime}$.

Suppose first that $\rho$ is strongly similar to $\Pi$, and let $u \in V(G)$. Then $\rho$ can be arrived at from $\Pi$ by a rearrangement of consecutive right- and left-hand endpoints so $r(\Pi u)$ and $r(\Pi(u \sigma))$ lie in a sequence of consecutive right-hand endpoints. Similarly $l(\Pi u)$ and $l(\Pi(u \sigma))$ lie in a sequence of consecutive left-hand endpoints so $u$ and $u \sigma$ have exactly the same neighbors in $G$. Since $G$ is reduced, we have $u=u \sigma$ and since $u$ was chosen arbitrarily, $\sigma$ is the identity. So we have shown that any nontrivial automorphism of $G$ carries $\Pi$ to a representation which is strongly similar to $\Pi^{\prime}$.

Suppose $\sigma$ and $\eta$ are nontrivial automorphisms of $G$. Then for $u, v \in V(G)$ we have

$$
\Pi u<\Pi v \text { iff } \Pi(u \sigma)>\Pi(v \sigma)
$$

and

$$
\Pi u<\Pi v \text { iff } \Pi(u \eta)>\Pi(v \eta) .
$$

Thus $\Pi u<\Pi v$ iff $\Pi(u(\sigma \eta))<\Pi(v(\sigma n))$ and so $\sigma \eta=1$. It follows that if $G$ has any nontrivial automorphisms then $G$ has automorphism group of size two.

It remains to show that if $G$ has a symmetric representation then $G$ has a nontrivial automorphism. The construction of such an automorphism is an easy exercise and is left to the reader. 
COROllary 2. Let $G$ be a dependable interval graph. Then either

(a) $G$ has no symmetric representations and the automorphism group of $G$ is a direct product of symmetric groups.

(b) Every representation of $G$ is symmetric and the automorphism group of $G$ is the semidirect product of $S_{2}$ with a direct product of symmetric groups.

Proof. This follows easily from Theorem 3 and the observation that any automorphism of $G$ induces an automorphism of $v(G)$.

4. Counting interval graphs. In this section we employ standard generating function techniques to first count the number of reduced interval graphs and then to count, for each reduced interval graph $D$, the number of connected interval graphs $G$ with $\mathfrak{v}(G)=D$.

Let $\delta_{n}$ denote the family of all $n$-sets of closed, real intervals having distinct endpoints chosen from the set $\{1,2, \ldots, 2 n\}$. Recall that two such sets are similar if one is obtained from the other by a rearrangement of consecutive endpoints and (perhaps) a flip of $\mathbf{R}$ over the point $n+\frac{1}{2}$. Our first job will be to count the similarity classes of $\delta_{n}$. To do so, we will view the flip of $\mathbf{R}$ across $n+\frac{1}{2}$ as the generator of a group of order 2 and apply Burnside's Lemma. This motivates the next definition.

DEFINITION 6. An ordered interval scheme of order $n$ is an equivalence class of $\delta_{n}$ where we call two sets of intervals equivalent if one is obtained from the other by a rearrangement of consecutive endpoints. An equivalence class which is (setwise) fixed when $\mathbf{R}$ is flipped across $n+\frac{1}{2}$ is called symmetric.

We want to add the number of ordered interval schemes to the number of symmetric ordered interval schemes and divide by 2 .

Ordered interval schemes are just interval graphs with certain automorphisms suppressed, and so the structural decomposition which we developed in $\$ \S 2$ and 3 for interval graphs is equally valid for interval schemes. In particular, we will talk of dependable and reduced schemes, maximal buried subschemes, and the dependable image of a scheme. To simplify our equations, we will begin by counting reduced interval schemes.

If $S$ is a set of intervals in an ordered interval scheme $G$, then $K(S)$ will denote the set of intervals of $G$ which intersect each interval in $S$.

Definition 7. Let $G$ be a connected interval scheme and $I$ an interval in $G$. $I$ is called a stone if the subscheme of $G$ induced by $K(\{I\})$ is complete (that is, if each pair of intervals in $K(\{I\})$ intersect). An interval in $G$ which is not a stone is called a pedestrian interval.

Recall that if $S$ is a subscheme of $G$ which contains two nonintersecting intervals then $K(S)$, if nonempty, is complete. So if $G$ is the dependable image of a connected, reduced interval scheme $H$, and if $I=I_{B}$ is the interval put in $G$ to replace a buried subscheme $B$ of $H$, then $I$ must be a stone. Moreover, any scheme $H$ obtained by replacing the stones of $G$ by nonempty reduced interval schemes will be a connected, reduced interval scheme whose dependable image is $G$. We can translate this into the language of generating functions as follows. 
Let $T(x, y)=\sum_{m, n} t_{m, n} x^{m} y^{n}$ where $t_{m, n}$ is the number of interval schemes with $m$ stones and $n$ pedestrian intervals and with the property that no two adjacent intervals have exactly the same neighbors. An isolated interval is deemed a stone and the empty scheme is not allowed.

Let $V(x, y)=\Sigma_{m, n} V_{m, n} x^{m} y^{n}$ where $v_{m, n}$ is the number of reduced schemes with $m$ stones and $n$ pedestrian intervals; the scheme with one interval is not counted.

Let $N(x, y)=\Sigma_{m, n} n_{m, n} x^{m} y^{n}$ where $n_{m, n}$ is the number of connected interval schemes with $m$ stones and $n$ pedestrian intervals and with the property that no two adjacent intervals have exactly the same neighbors. Note that

$$
T(x, y)=N(x, y) /(1-N(x, y))
$$

and so

$$
N(x, y)=T(x, y) /(1+T(x, y)) .
$$

If $G$ is a reduced interval scheme with $m$ stones and $n$ pedestrian intervals then the generating function for the set of all reduced interval schemes $H$ with $\mathfrak{b}(H)=G$ is

$$
y^{n}(T(x, y))^{m} .
$$

Let $T^{(1)}(x, y)$ denote the generating function for reduced interval schemes which have at least 2 intervals and which have an interval intersecting every other interval. We have $T^{(1)}(x, y)=y\left(T(x, y)-x-T^{(1)}(x, y)\right)$ or

$$
T^{(1)}(x, y)=\left(\frac{y}{1+y}\right)(T(x, y)-x) .
$$

Each connected, reduced interval scheme $D$ must fall into one of the 3 categories below.

(a) $\mathfrak{b}(D)=G$ for some reduced scheme $G$.

(b) $D$ has at least 2 intervals and an interval which intersects every other interval.

(c) $D$ has one point.

The generating function for all connected, reduced schemes $D$ in category (a) is (4.2) summed over all reduced schemes $G$. This is seen to be $V(T(x, y), y)$ and so we are led to the equation

$$
N(x, y)=V(T(x, y), y)+(y /(1+y))(T(x, y)-x)+x .
$$

Rewriting the above we have

$$
V(T(x, y), y)=\frac{-x}{(1+y)}-\left(\frac{y}{1+y}\right) T(x, y)+\frac{T(x, y)}{1+T(x, y)} .
$$

Consider the $\mathbf{C}$-algebra homomorphism $\psi: \mathbf{Q}[[x, y]] \rightarrow \mathbf{Q}[[x, y]]$ given by

$$
\psi(x)=T(x, y), \quad \psi(y)=y .
$$

Note that $T(x, y)=x+T_{2}(x, y)$ where $T_{2}(x, y)$ is a series in which every term $x^{a} y^{b}$ has order 2 or more (the order of $x^{a} y^{b}$ is $a+b$ ). In view of this fact, it is seen that $\psi$ is an isomorphism and so has a unique inverse. Of course $\psi^{-1}(y)=y$ and $\psi^{-1}(x)$ is the generating function $F(x, y)$ which satisfies $F(0,0)=0$ and $x=$ $T(F(x, y), y)$ or

$$
F(x, y)=x-T_{2}(F(x, y), y) .
$$


In particular, $T(x, y)$ determines $F(x, y)$ recursively. We may express (4.4) in terms of $\psi$ as

$$
\psi(V(x, y))=\psi\left(\frac{-F(x, y)}{1+y}-\frac{x y}{1+y}+\frac{x}{1+x}\right)
$$

Applying $\psi^{-1}$ we have

$$
V(x, y)=\frac{-F(x, y)}{1+y}-\frac{x y}{1+y}+\frac{x}{1+x} .
$$

Recall that $V(x, y)$ is the generating function which we want to compute. Together, equations (4.5) and (4.6) show that if $T(x, y)$ is known, then $V(x, y)$ can be determined from it. We turn our attention to the problem of computing $T(x, y)$.

Let $H$ be a reduced interval scheme and let $r(H)$ denote the interval in $H$ whose left-hand endpoint sits furthest to the right. Consider the interval scheme $H-r(H)$ obtained by removing $r(H)$ from $H$. Unfortunately, $H-r(H)$ may not be reduced for it may be that in $H$ there exist two intervals $u$ and $v$ which have exactly the same neighbors except that $u$ intersects $r(H)$ and $v$ does not. We identify all such pairs in $H-r(H)$ to obtain a reduced interval scheme $G$ of strictly smaller size than $H$ called the predecessor of $H$. We now examine the reverse process of creating from $G$ reduced, interval schemes $H$ having $G$ as their predecessor.

We call an interval $I$ in $G$ an end-interval if $I$ intersects $r(G)$, but is not itself $r(G)$. Suppose $G$ has $l$ stones, $m$ pedestrian intervals, $n$ end-intervals and the interval $r(G)$. We give $G$ the weight $x^{l} y^{m} z^{n}$ noting that $l+m+n+1$ is the number of intervals in $G$.

We add a new interval $r(H)$ to the right of $G$ with the intent of creating all reduced interval schemes $H$ having $G$ as their predecessor. Consider what can happen to the various intervals in $G$. We have the following 3 possibilities:

(a) $r(G)$ can intersect $H$ and become an end-interval in $H$ with weight $z$.

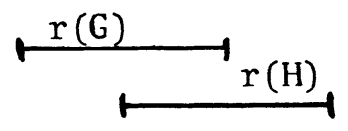

(b) $r(G)$ can fall short of intersecting with $r(H)$ and become a stone in $H$ with weight $x$.

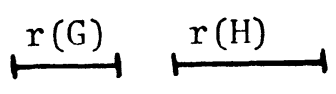

(c) $r(G)$ can split into two intervals, one becoming an end-interval, total weight $x z$.

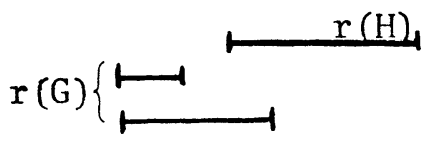


Each end-interval $E$ of $G$ has three similar possibilities:

(a) $E$ can intersect $H$ and become an end-interval in $H$ with weight $z$.

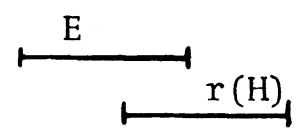

(b) $E$ can fall short of intersecting with $r(H)$ and become a pedestrian interval in $H$ with weight $y$.

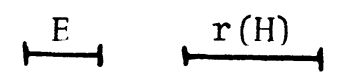

(c) $E$ can split into two intervals, one becoming a pedestrian interval and one becoming an end-interval with total weight $y z$.

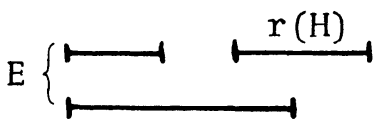

Of course the stones and pedestrian intervals of $G$ must remain as they are since they do not have endpoints extending beyond the right-hand side of $G$. Every possible combination of choices for $r(G)$ and for the end-intervals of $G$ creates a reduced interval scheme with one exception; if $r(G)$ does not become a stone and if every end-interval intersects $r(H)$ without splitting, then $r(G)$ and $r(H)$ have exactly the same neighbors and the resulting scheme is not reduced. So the generating function for all reduced interval schemes $H$ having $G$ as their predecessor is

$$
(x+z+x z) x^{l} y^{m}(y+z+y z)^{n}-x^{l} y^{m} z^{n+1} .
$$

Let $\eta(x, y, z)=\Sigma_{l, m, n} w(l, m, n) x^{l} y^{m} z^{n}$ where $w(l, m, n)$ is the number of reduced interval schemes $G$ with $l$ stones, $m$ pedestrian intervals, $n$ end-intervals and $r(G)$. Then

(4.7) $\eta(x, y, z)=1+(x+z+x z) \eta(x, y,(y+z+y z))-z \eta(x, y, z)$.

The initial condition 1 accounts for the scheme with only one interval. This equation recursively defines $\eta(x, y, z)$ and $T(x, y)$ can be easily obtained from $\eta(x, y, z)$ by observing that in $G$, each end-interval is a pedestrian interval and that $r(G)$ is a stone. Thus we have

$$
T(x, y)=x \eta(x, y, y)
$$

which solves the problem of computing $T(x, y)$.

A similar process will be used to obtain the generating function for reduced symmetric schemes. In this case however, the intervals come in two varieties; those that are fixed when $\mathbf{R}$ is flipped over $n+\frac{1}{2}$ (called symmetric intervals) and those that fall into 2-cycles when $\mathbf{R}$ is flipped over $n+\frac{1}{2}$. A stone which is symmetric must necessarily sit directly in the middle of our scheme, having endpoints $n$ and $n+1$. Hence for every scheme $G$ with no symmetric stone there is a unique dual scheme $\bar{G}$ 
which is identical to $G$ except that a symmetric stone has been inserted in the middle. We will only count schemes in which there is no symmetric stone and later account for the dual schemes.

Let $V_{s}(u, x, y)=\Sigma_{k, l, m} v_{s}(k, l, m) u^{k} x^{l} y^{m}$ where $v_{s}(k, l, m)$ is the number of reduced symmetric interval schemes with $k$ symmetric pedestrian intervals, $l 2$-cycles of stones and $m$ 2-cycles of pedestrian intervals.

In particular, the total number of intervals in a scheme counted by $v_{s}(k, l, m)$ is $k+2 l+2 m$. Define the series $N_{s}(u, x, y)$ and $T_{s}(u, x, y)$ analogously for connected, reduced interval schemes and arbitrary reduced interval schemes. It is worth noting that $N_{s}(u, x, y)$ and $T_{s}(u, x, y)$ are related by

$$
T_{s}(u, x, y)=\left(1+N_{s}(u, x, y)\right)(1+T(x, y))-1 \text {. }
$$

This relationship is obtained as follows. The connected components of a symmetric interval scheme $\alpha$ can be divided into two sets depending on whether they fall in a 1-cycle or a 2-cycle when the real line is flipped over $n+\frac{1}{2}$ ( $n$ being the number of intervals of $\alpha$ ). Any connected component which is fixed must sit in the middle of $\alpha$ and so there can be at most one such component. If one exists, it must be a connected symmetric interval scheme. Thus the factor of $\left(1+N_{s}(u, x, y)\right)$ accounts for the possible existence of a symmetric connected component.

The remaining components fall in 2-cycles. Let $\beta$ be the scheme given by the components which sit above the point $n+\frac{1}{2}$. A mirror image of $\beta$ sits below $n+\frac{1}{2}$ and every point above creates a 2-cycle with its image below. In total these intervals contribute a weight of $x^{r} y^{s}$ to the weight of $\alpha$ where $r$ is the number of stones of $\beta$ and $s$ is the number of pedestrian intervals of $\beta$. Summing this over all possible $\beta$ gives $T(x, y)$; we multiply by $(1+T(x, y))$ to allow for the fact that $\alpha$ may consist of only a symmetric connected component.

Rewriting this relationship we have

$$
N_{s}(u, x, y)=\frac{1+T_{s}(u, x, y)}{1+T(x, y)}-1 .
$$

Equation (4.9) will be of use later.

Let $T^{(1)}(u, x, y)$ denote the generating function for symmetric reduced interval schemes which have an interval intersecting every other interval. We have $T_{s}^{(1)}(u, x, y)=u\left(T_{s}(u, x, y)-T_{s}^{(1)}(u, x, y)\right)$ and so

$$
T_{s}^{(1)}(u, x, y)=\left(\frac{u}{1+u}\right)\left(T_{s}(u, x, y)\right)
$$

As before we obtain

$$
N_{s}(u, x, y)=V_{s}(u, T(x, y), y)+(u /(1+u))\left(T_{s}(u, x, y)\right)+x
$$

and so

$$
V_{s}(u, x, y)=\frac{T_{s}(u, F(x, y), y)}{1+x}-\left(\frac{u}{1+u}\right) T_{s}(u, F(x, y), y)-F(x, y) .
$$

Note that the inverting series $F(x, y)$ is the same one used before and so need not be recomputed. This equation reduces our problem to that of computing $T_{s}(u, x, y)$. 
We compute $T_{s}(u, x, y)$ recursively in almost the same way we did $T(x, y)$. This time $r(G)$ and $r(H)$ will indicate the 2-cycle consisting of the interval whose left-hand endpoint sits furthest to the right and the interval whose right-hand endpoint sits furthest to the left. A symmetric endpoint, if one exists, is not indicated by a variable $z$. Such an endpoint must intersect every interval in our scheme, hence must be unique. We therefore have no need of indicating how many of them there are-we simply allow for the possibility that it is there when performing the recursion. Such a symmetric endpoint $E$ has the following range of possibilities;

(a) It does not exist-weight 1 .

(b) It fails to intersect the cycle at both ends and becomes a symmetric pedestrian interval with weight $u$.

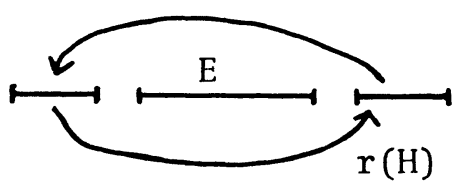

(c) It splits into two end-intervals; one an end-interval on the right side and one an interval on the left side. Weight $z$.

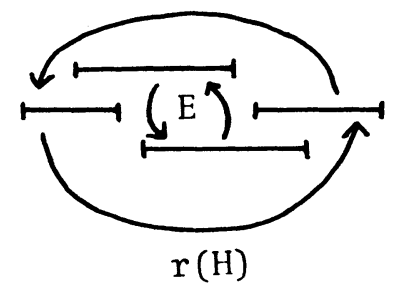

(d) It does both (b) and (c).

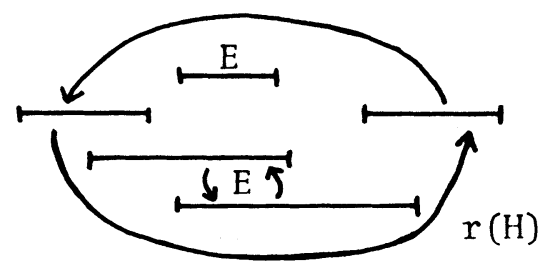

$E$ may likewise become a symmetric end-interval and do any of (a), (b), (c) or (d) but these possibilities are ignored since at the next recursion step we will be allowing for both the existence and nonexistence of a symmetric end-interval.

The range of possibilities for $r(G)$ and for the other end-intervals are exactly as before. Let $\sigma(u, x, y, z)=\Sigma_{k, l, m, n} w_{s}(k, l, m, n) u^{k} x^{l} y^{m} z^{n}$ where $w_{s}(k, l, m, n)$ is the number of symmetric reduced interval schemes with $k$ symmetric pedestrian intervals, $l$ 2-cycles of stones, $m$ 2-cycles of pedestrian intervals and $n 2$-cycles of 
end-intervals. We are led to the following equation which recursively defines $\sigma(u, x, y, z)$;

$$
\begin{aligned}
\sigma(u, x, y, z)= & (1+z)+(1+u+z+u z)(x+z+x z) \sigma(u, x, y,(y+z+y z)) \\
& -(1+z) z \sigma(u, x, y, z) .
\end{aligned}
$$

Here the initial conditions $(1+z)$ indicate the schemes

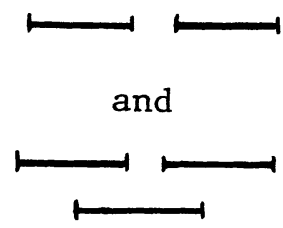

1

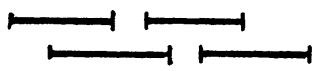

and

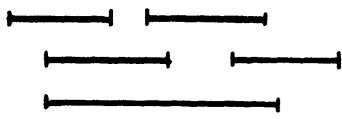

z

From $\sigma(u, x, y, z)$ we derive the series $T_{s}(u, x, y)$ by

$$
T_{s}(u, x, y)=(1+u) x \sigma(u, x, y, y) .
$$

The factor $(1+u)$ in (4.12) allows for the possibility of a symmetric endpoint. This solves the problem of computing $T_{s}(u, x, y)$ which in turn solves the problem of computing $V_{s}(u, x, y)$.

Recall that $V(x, y)$ and $V_{s}(u, x, y)$ count reduced interval schemes and symmetric reduced interval schemes. We have just shown how to obtain each of these series.

This corollary allows us to derive immediately the generating functions for dependable and reduced interval graphs from the series $V(x, y)$ and $V_{s}(u, x, y)$.

Let $D(y)=\sum_{n=1}^{\infty} d_{n} y^{n}$ where $d_{n}$ is the number of dependable interval graphs on $n$ points.

Let $W(y)=\sum_{n=1}^{\infty} w_{n} y^{n}$ where $w_{n}$ is the number of reduced interval graphs on $n$ points.

$$
\begin{aligned}
& D(y)=\frac{1}{2}\left(V\left(\frac{y}{1-y}, \frac{y}{1-y}\right)+\left(\frac{1}{1-y}\right) V_{s}\left(\frac{y}{1-y}, \frac{y}{1-y^{2}}, \frac{y^{2}}{1-y^{2}}\right)\right), \\
& W(y)=\frac{1}{2}\left(V(y, y)+(1+y) V_{s}\left(y, y^{2}, y^{2}\right)\right) .
\end{aligned}
$$

We now turn our attention to the main problem-that of counting interval graphs. Our process for counting interval graphs naturally breaks into two halves-we have currently completed the first half. This involved counting ordered interval schemes (where buried subschemes are allowed no automorphisms),

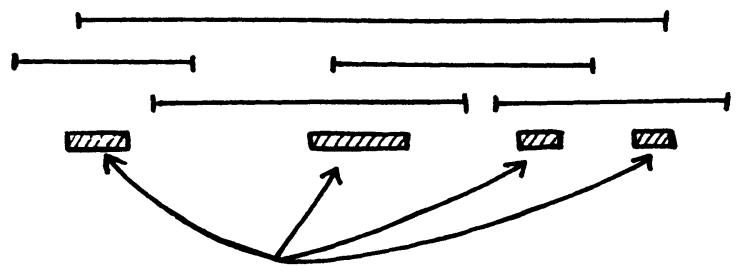

buried subschemes 
and then removing the buried subschemes leaving stones behind to mark where they were.

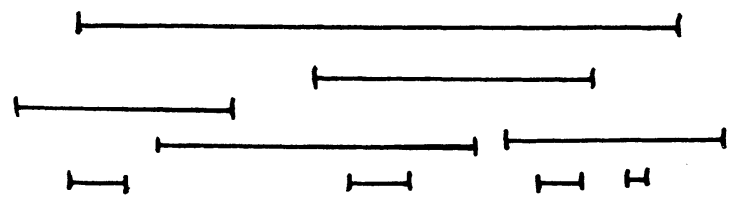

We will now replace them but this time as interval graphs (with all allowable automorphisms).

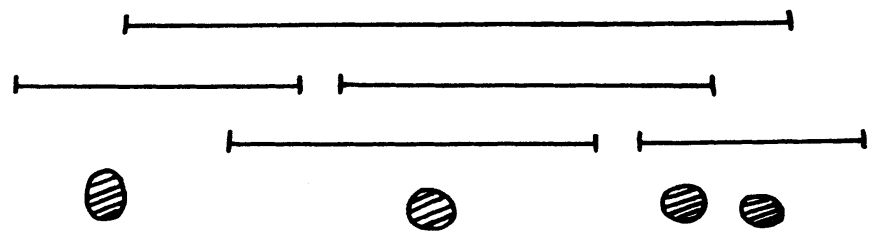

This step is accomplished merely by substituting for each stone indicator $x$ the generating function, $I(y)$, for interval graphs.

Let $I(y)=\sum_{n=1}^{\infty} i_{n} y^{n}$ and $C(y)=\sum_{n=1}^{\infty} c_{n} y^{n}$ where $i_{n}$ is the number of interval graphs with $n$ points and $c_{n}$ is the number of connected interval graphs with $n$ points.

THEOREM 3. The series $I(y)$ and $C(y)$ are related by the equations (1) and (2) below.

$$
\begin{gathered}
I+I(y)=\exp \left(\sum_{k=1}^{\infty} \frac{C\left(y^{k}\right)}{k}\right) . \\
C(y)=y(1+I(y))+\frac{1}{2} V\left(I(y), \frac{y}{1-y}\right) \\
+\frac{1}{2}(1+I(y)) V_{s}\left(\frac{y}{1-y}, I\left(y^{2}\right), \frac{y^{2}}{1-y^{2}}\right) .
\end{gathered}
$$

Proof (of (1)). This follows by a standard application of Pólya's Theorem. For details see Harary and Palmer [11, p. 90].

(OF (2)). The initial term $y(1+I(y))$ accounts for all interval graphs which have one interval intersecting every other interval. Any interval graph $G$ not of that form has a reduced image $\nu(G)$ and $G$ is obtained from $\nu(G)$ by replacing each stone of $\mathfrak{v}(G)$ with an interval graph.

The $\frac{1}{2}$ accounts for the fact that any such interval graph $G$ having an automorphism which induces a flip of $\mathfrak{v}(G)$ is counted once in $B(I(y), y /(1-y))$ and once in $(1+I(y)) V_{s}\left(y /(1-y), I\left(y^{2}\right), y^{2} /\left(1-y^{2}\right)\right)$ whereas all other such interval graphs are counted twice in $V(y /(1-y), I(y))$.

Note that using equations (1) and (2), $I(y)$ and $C(y)$ can be obtained recursively from $V(x, y)$ and $V_{s}(x, y)$. Tables of $i_{n}$ and $c_{n}$ can be found in $\S 9$ for $n \leqslant 30$. 
5. Counting other sorts of interval graphs. In this section we count labelled graphs which are interval graphs, interval graphs with automorphism group of size 1, and interval graphs with a transitive automorphism group.

Let $L(y)=\sum_{n=1}^{\infty}\left(l_{n} y^{n} / n !\right)$ and $C_{L}(y)=\sum_{n=1}^{\infty}\left(c_{n}^{L} y^{n} / n !\right)$ be the exponential generating functions for labelled interval graphs and connected, labelled interval graphs respectively.

THEOREM 4. The series $L(y)$ and $C_{L}(y)$ are related by equations (1) and (2) below.

$$
\begin{gathered}
1+L(y)=\exp \left(C_{L}(y)\right) . \\
C_{L}(y)=\left(1-e^{-y}\right)(1+L(y))+\frac{1}{2} V\left(L(y),\left(e^{y}-1\right)\right) .
\end{gathered}
$$

Proof. Equation (1) is arrived at using standard arguments about exponential generating functions. See [11, p. 8]. We now prove (2).

Consider first, the set $L^{(1)}$ of labelled connected interval graphs having one vertex adjacent to every other. If $G \in L^{(1)}$ and $S$ is the set of vertices in $G$ which are adjacent to every vertex in $G$ then the subgraph $G-S$ is either empty (in which case $G$ is complete) or is a labelled interval graph which does not have one vertex adjacent to every other.

Let $L^{(1)}(y)$ be the exponential generating function for the set $L^{(1)}$. The exponential generating function for all complete graphs is $e^{y}-1$. The exponential generating function for all labelled interval graphs which do not have one vertex adjacent to every other is $L(y)-L^{(1)}(y)$. If $H$ is such a graph and has $h$ points then the exponential generating function for all graphs $G$ obtained from $H$ by adding $s$ points adjacent to each other and to every point in $H$ is

$$
\frac{1}{(s+h) !}\left(\begin{array}{c}
s+h \\
s
\end{array}\right) y^{s+h}=\frac{y^{s} y^{h}}{s ! h !} .
$$

Summing this over all $H$ and all $s$ we obtain the series $\left(e^{y}-1\right)\left(L(y)-L^{(1)}(y)\right)$. It follows that

$$
L^{(1)}(y)=\left(e^{y}-1\right)+\left(e^{y}-1\right)\left(L(y)-L^{(1)}(y)\right) .
$$

Rewriting this equation we get $L^{(1)}(y)=\left(1-e^{-y}\right)(1+L(y))$. This accounts for the first factor in equation (2).

By Corollary 1, a reduced interval graph has automorphism group of size 1 or 2 depending on whether it is given weight 1 or $\frac{1}{2}$ in the generating function $\frac{1}{2} V(x, y)$. Since the number of ways to label a graph on $n$ points is $n$ ! divided by the order of its automorphism group we see that $\frac{1}{2} V(y, y)$ is the exponential generating function for labelled, reduced interval graphs.

Suppose that $D$ is a labelled, reduced interval graph with $n$ stones and $m$ pedestrian points. Then the exponential generating function for the set of labelled, connected interval graphs $G$ with $\mathfrak{b}(G)=D$ is $L(y)^{n}\left(e^{y}-1\right)^{m}$. Summing this over all labelled, reduced interval graphs we obtain $\frac{1}{2} V\left(L(y), e^{y}-1\right)$.

Equations (1) and (2) recursively define $L(y)$ and $C_{L}(y)$. The values of $l_{n}$ and $c_{n}^{L}$ for $n \leqslant 25$ appear in $\$ 9$. 
We next consider identity interval graphs, these being interval graphs which have a trivial automorphism group. Let $J(y)=\sum_{n=1}^{\infty} j_{n} y^{n}$ and $K(y)=\sum_{n=1}^{\infty} k_{n} y^{n}$ where $j_{n}$ is the number of identity interval graphs with $n$ points and $k_{n}$ is the number of connected, identity interval graphs with $n$ points.

THEOREM 5. The series $J(y)$ and $K(y)$ are related by equations (1) and (2) below.

$$
\begin{aligned}
1+ & J(y)=\exp \left(-\sum_{l=1}^{\infty} \frac{(-1)^{l} K\left(y^{l}\right)}{l}\right) . \\
K(y)= & \left(\frac{y}{1+y}\right)(1+J(y))+\frac{1}{2} V(J(y), y) \\
& -\frac{1}{2}(1+J(y)) V_{s}\left(y, J\left(y^{2}\right), y^{2}\right) .
\end{aligned}
$$

Proof. Equation (1) is arrived at by standard arguments. See Pólya [14]. We now prove (2).

Let $J^{(1)}(y)$ denote the generating function for identity interval graphs which have one point adjacent to all others. If such a point exists in an identity interval graph $G$, it is unique for if $G$ has two such points then the interchange of these points would constitute a nontrivial automorphism of $G$. Let $G$ be an identity interval graph having a point $v$ which is adjacent to every other point of $G . G-v$ is either empty (in which case $G$ is the one point graph) or $G-v$ is an identity interval graph having no vertex adjacent to every other. It follows that $J^{(1)}(y)=y+y\left(J(y)-J^{(1)}(y)\right)$. Rewriting this we obtain

$$
J^{(1)}(y)=(y /(1+y))(1+J(y))
$$

which accounts for the first factor in equation (2).

Suppose $D$ is a reduced interval graph, and let $G$ be a connected interval graph with $\mathfrak{v}(G)=D$. If $G$ is an identity graph then $G$ was obtained from $D$ by replacing each stone with an identity interval graph. Conversely, if $G$ is obtained from $D$ by replacing each stone with an identity interval graph then either $G$ is an identity interval graph or $D$ is symmetric, the same identity graph replaces opposite pairs of stones and the automorphism group of $G$ has size 2 . We see that the generating function for all such connected interval graphs having no point adjacent to every other point is $\frac{1}{2}\left(V(J(y), y)+(1+J(y)) V_{s}\left(y, J\left(y^{2}\right), y^{2}\right)\right)$.

A similar problem is to count all interval graphs whose automorphism group acts transitively on their vertex set. Such graphs are called transitive interval graphs. Clearly, a graph is transitive iff all of its connected components are isomorphic and each is transitive. A connected transitive interval graph having one point adjacent to every other is obviously complete. These are the only transitive, connected interval graphs because any other must have a dependable image fixed under every automorphism. But a dependable interval graph can never be transitive since such a dependable interval graph would necessarily have a transitive reduced image contrary to Corollary 1. So the only transitive interval graphs are disjoint unions of $K_{n}$, 
the complete graph on $n$ points. Their generating function is given by

$$
\sum_{n=1}^{\infty} 1 /\left(1-y^{n}\right) \text {. }
$$

It is worth noting that bridgeless and nonseparable interval graphs can be counted though to do so requires that a new variable be introduced into the series $V(x, y)$ which keeps track of left-hand endpoints.

Circular arc graphs (see Klee [13]) can also be counted though this enumeration requires slightly different techniques and will appear elsewhere.

6. Unit interval graphs. A graph $G$ is a unit interval graph if it has an interval representation $\Pi=\left\{\Pi v_{1}, \ldots, \Pi v_{n}\right\}$ in which each interval $\Pi v_{i}$ has length 1 . Such a representation is called a unit representation. Not every interval graph is a unit interval graph; in particular the complete bipartite graph $K_{1,3}$ is an interval graph but not a unit interval graph. In this section we count unit interval graphs, labelled unit interval graphs and identity unit interval graphs. Fred Roberts [17], has characterized unit interval graphs in terms of forbidden subconfigurations though we will use the tools developed in $\S \S 2$ and 5 to perform the enumeration. My thanks to Professor Frank Harary for suggesting this problem.

Almost every connected unit interval graph is dependable. In fact there is one infinite family of exceptions. Recall that an interval graph is reduced if it is connected and has no two adjacent points with exactly the same neighbors. Define the reduced unit interval graphs $S_{n}, n=0,1,2, \ldots$, to be the graphs given by the representations $\sigma_{n}$ below:

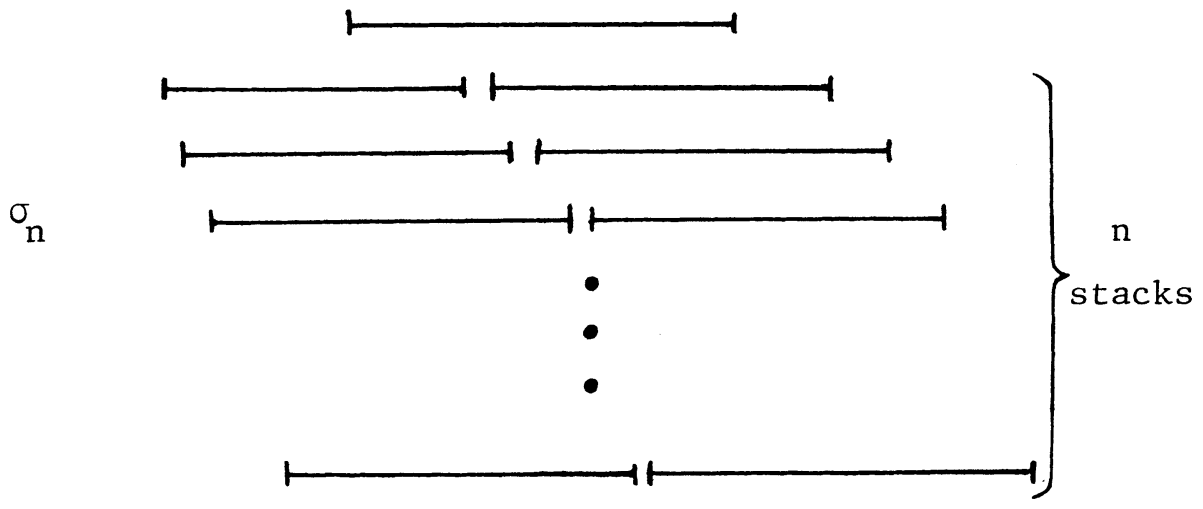

LemMa 4. Let $G$ be a reduced unit interval graph which is not dependable. Then $G=S_{n}$ for some $n$.

Proof. Since $G$ is not dependable, $G$ has a buried subgraph $B$. We first show that $V(G)-G-K(B)$ is empty. Suppose to the contrary that $V(G)-B-K(B) \neq \varnothing$; choose $v \in V(G)-B-K(B)$ such that $v$ is adjacent to some point $\omega$ of $K(B)$ (this can be done since $G$ is connected). The set $B$ contains two nonadjacent points $b_{1}$ and $b_{2}$ both of which are adjacent to $\omega$ and nonadjacent to $v$. The subgraph of $G$ induced 
by $\left\{v, \omega, b_{1}, b_{2}\right\}$ is isomorphic to $K_{1,3}$ which is a contradiction. So $V(G)-B-$ $K(B)=\varnothing$. Next we observe that $K(B)$ contains only one point $k$, as $G$ is reduced, and that $B$ does not contain three mutually nonadjacent points.

Let $\Pi$ be an interval representation of $G$ and let $A=\{b \in B: \Pi v<\Pi b$ for some $v \in V(G)\}$. Number the points in $A$ as $b_{1}, \ldots, b_{n}$ so that $l\left(\Pi b_{1}\right)<l\left(\Pi b_{2}\right)<\cdots<$ $l\left(\Pi b_{n}\right)$.

Let $C_{1}, \ldots, C_{n}$ be defined by $C_{i}=\left\{v \in B: \Pi v<\Pi b_{i}\right\}$. Note that

(1) $A$ is nonempty since $B$ is not complete.

(2) $A$ induces a complete subgraph of $G$ since $B$ has no 3 mutually nonadjacent points.

(3) $C_{i} \neq 0$ for all $i$ by the definition of $A$.

(4) $C_{i}$ induces a complete subgraph of $G$ since $B$ contains no 3 mutually nonadjacent points.

(5) $C_{i+1}-C_{i} \neq \varnothing$ since otherwise, $b_{i}$ and $b_{i+1}$ would have exactly the same neighbors.

(6) $V(G)=\{k\} \cup A \cup C_{n}$.

(7) If $u$ is a point in $C_{i+1}-C_{i}$ then $u$ has as neighbors in $G$ exactly the points in the set $(V(g)-A) \cup\left\{b_{1}, \ldots, b_{i}\right\}$.

From (7) it follows that any two points in $C_{i+1}-C_{i}$ have exactly the same neighbors so $C_{i+1}-C_{i}$ must contain precisely one point. It is now clear that $G=S_{n}$, which completes the proof of Lemma 4.

It is easy to see that $S_{n}$ has only one representation up to similarity. Combining this fact with Lemma 4 and Theorem 1 we have the following.

LeMma 5. Let $G$ be a reduced unit interval graph. Then $G$ has one representation up to similarity.

Something much stronger is true. Observe that the graph $S_{n}$ has exactly one unit representation up to the equality of representations introduced in $\S 1$. In fact, reduced unit interval graphs have no more than 2 unit representations. In order to prove this we need the following technical lemma.

LeMma 6. Let $\Pi$ and $\rho$ be unit representations of the reduced unit interval graph $G$. Suppose that for all pairs of points $u$ and $v$ we have $\Pi u<\Pi v$ iff $\rho u<\rho v$. Then for all pairs of points $u$ and $v$ we have $r(\Pi u)<r(\Pi v)$ iff $r(\rho u)<r(\rho v)$.

Proof. Suppose to the contrary that there exists points $u$ and $v$ with $r(\Pi u)<$ $r(\Pi v)$ and $r(\rho u)<r(\rho v)$. By Lemma 5, we can obtain $\rho$ from $\Pi$ by a rearrangement of consecutive left- and right-hand endpoints. In doing so we must interchange the right-hand endpoints of $\Pi u$ and $\Pi v$ and so $r(\Pi u)$ and $r(\Pi v)$ must lie in a sequence of consecutive right-hand endpoints. Hence there exists no interval $\Pi(w)$ with $r(\Pi u)<l(\Pi w)<r(\Pi v)$. When obtaining $\rho$ from $\Pi$ we must interchange the left-hand endpoints of $\Pi u$ and $\Pi v$ as well since both $\Pi$ and $\rho$ are unit representations. So $l(\Pi u)$ and $l(\Pi v)$ lie in a sequence of consecutive left-hand endpoints. Thus there is no point $w$ with $l(\Pi u)<r(\Pi w)<l(\Pi v)$. Hence $u$ and $v$ have the same neighbors which contradicts $G$ being reduced. 
THEOREM 6. Let $G$ be a reduced unit interval graph. Then $G$ has exactly one or two unit representations depending on whether $G$ is or is not symmetric.

Proof. Let $\Pi$ and $\rho$ be unit representations of $G$. By Lemma 5, $\Pi$ and $\rho$ are similar so either (a) $\Pi u<\Pi v$ iff $\rho v<\rho v$ for all $u, v \in V(G)$ or (b) $\Pi u<\Pi v$ iff $\rho v<\rho u$ for all $u, v \in V(G)$. If (a) is the case, let $\bar{\rho}=\rho$; if (b) is the case let $\bar{\rho}$ be the opposite of $\rho$. Applying Lemma 6 we see that $\bar{\rho}=\Pi$ and the theorem follows.

An ordered, unit interval scheme is reduced if it represents a reduced unit interval graph. Theorem 6 tells us that if we have counted reduced, ordered, unit interval schemes up to a flip of $\mathbf{R}$ then we have counted reduced unit interval graphs. We proceed as in $\$ 4$, viewing the flip of $\mathbf{R}$ as generating a group of order 2 which acts on the set of reduced, ordered unit interval schemes. As before we will apply Burnsides' Lemma; we add the total number of reduced, unit interval schemes to the number of symmetric, reduced unit interval schemes and divide by 2 .

We say that the reduced unit interval scheme $P$ is the predecessor to the reduced unit interval scheme $Q$ if $G$ is obtained from $Q$ by removing the right-most interval from $G$ and then identifying pairs of intervals with exactly the same neighbors.

LEMMA 7. Let $P$ be a reduced, unit interval scheme with $r$ intervals and $s$ end-intervals. Then

(a) $P$ is the predecessor of exactly one reduced unit interval scheme with $(r+1)$ intervals and $i$ end-intervals for each $i=2,3, \ldots, s$.

(b) $P$ is the predecessor of exactly one reduced unit interval scheme with $(r+2)$ intervals and $i$ end-intervals for each $i=2,3, \ldots,(s+1)$.

(c) $P$ is the predecessor of no other reduced unit interval schemes.

Proof. Let $\varepsilon_{1}, \ldots, \varepsilon_{s}$ be the end-intervals of $P$. By Lemma 6 there is a natural ordering on their right-hand endpoints. Assume the $\varepsilon_{i}$ 's are numbered so that $r\left(\varepsilon_{1}\right)<r\left(\varepsilon_{2}\right)<\cdots<r\left(\varepsilon_{s}\right)$. If we add a new interval $\eta$ to the right of $P$, $\eta$ has the following $(2 s-1)$ options:

$$
\begin{gathered}
\text { Case (a) }\left\{\begin{array}{cc}
r\left(\varepsilon_{1}\right)<l(\eta)<r\left(\varepsilon_{2}\right) & s \text { end-intervals } \\
r\left(\varepsilon_{1}\right)<l(\eta)<r\left(\varepsilon_{3}\right) & (s-1) \text { end-intervals } \\
\vdots & \vdots \\
r\left(\varepsilon_{s-1}\right)<l(\eta)<r\left(\varepsilon_{s}\right) & 2 \text { end-intervals }
\end{array}\right. \\
\text { Case (b) }\left\{\begin{array}{cc}
r\left(\varepsilon_{1}^{L}\right)<l(\eta)<r\left(\varepsilon_{1}^{u}\right) & (s+1) \text { end-intervals } \\
r\left(\varepsilon_{2}^{L}\right)<l(\eta)<r\left(\varepsilon_{2}^{u}\right) & s \text { end-intervals } \\
\vdots & \vdots \\
r\left(\varepsilon_{s}^{L}\right)<l(\eta)<r\left(\varepsilon_{s}^{u}\right) & 2 \text { end-intervals }
\end{array}\right.
\end{gathered}
$$

The notation $r\left(\varepsilon_{i}^{L}\right)<l(\eta)<r\left(\varepsilon_{i}^{u}\right)$ indicating that the interval $\varepsilon_{i}$ has split in two with the left-hand endpoint of $\eta$ falling between the two right-hand endpoints. 


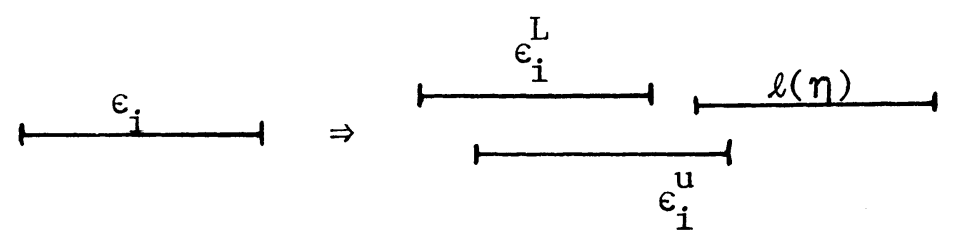

Thus we have verified (a) and (b), and (c) is obvious.

Let $a(r, s)$ denote the number of reduced, unit interval schemes with $r$ intervals and $s$ end-intervals. We will not count the scheme having only one interval. Lemma 7 gives us an easy method for generating the numbers $a(r, s)$. This recursion is given in the next theorem.

THEOREM 7. The numbers $a(r, s)$ satisfy the following recursion:

(a) $a(1, s)=a(2, s)=0$ for all $s$.

(b) $a(r, 1)=0$ for all $r$.

(c) $a(r, s)=0$ if $s>|(r+1) / 2|$.

(d) $a(3,2)=1$.

(e) $a(r, s)=\sum_{i=s}^{\infty} a(r-1, i)+\sum_{i=s-1}^{\infty} a(r-2, i)$ for $2 \leqslant s \leqslant|(r+1) / 2|$.

The numbers $a(r, s)$ for small $r$ and $s$ appear below.

\begin{tabular}{r|rrrrrr}
$r \backslash s$ & 2 & 3 & 4 & 5 & 6 & Total \\
\hline 1 & 0 & 0 & 0 & 0 & 0 & 0 \\
2 & 0 & 0 & 0 & 0 & 0 & 0 \\
3 & 1 & 0 & 0 & 0 & 0 & 1 \\
4 & 1 & 0 & 0 & 0 & 0 & 1 \\
5 & 2 & 1 & 0 & 0 & 0 & 3 \\
6 & 4 & 2 & 0 & 0 & 0 & 6 \\
7 & 9 & 5 & 1 & 0 & 0 & 15 \\
8 & 21 & 12 & 3 & 0 & 0 & 36 \\
9 & 51 & 30 & 9 & 1 & 0 & 91 \\
10 & 127 & 76 & 25 & 4 & 0 & 232 \\
11 & 323 & 196 & 69 & 14 & 1 & 603 \\
12 & 835 & 512 & 189 & 44 & 5 & 1585
\end{tabular}

TABLE I. $a(r, s)$

It is worth noting that the initial condition $a(3,2)=1$ accounts for the reduced, unit interval scheme

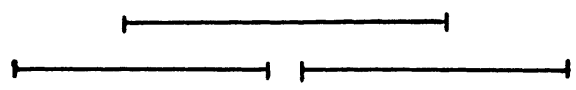

We move now to the symmetric case where the situation is more complicated. Here we form the predecessor $P$ of a reduced unit interval graph $Q$ by removing both the left-most interval $\lambda$ and the right-most interval $\rho$. If two intervals $\varepsilon_{1}$ and $\varepsilon_{2}$ 
have exactly the same neighbors in $Q$ except that $\varepsilon_{1}$ intersects $\rho$ and $\varepsilon_{2}$ does not, we identify $\varepsilon_{1}$ and $\varepsilon_{2}$ in $P$; similarly for two intervals $\varepsilon_{1}$ and $\varepsilon_{2}$ which have exactly the same neighbors except that $\varepsilon_{1}$ intersects $\lambda$ and $\varepsilon_{2}$ does not.

Note that the predecessor of a symmetric, reduced unit interval scheme may not be reduced. For example if

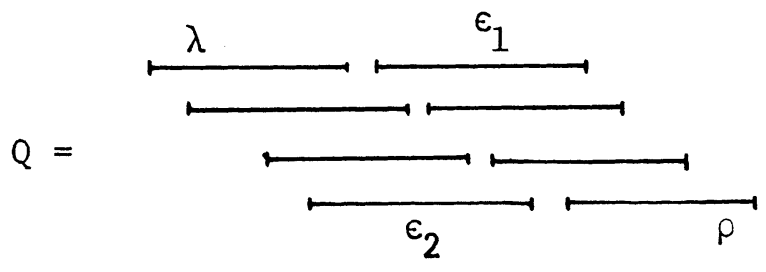

Then

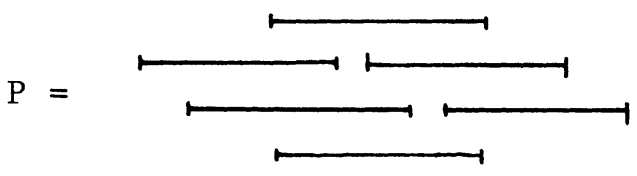

The problem is that there may be intervals $\varepsilon_{1}$ and $\varepsilon_{2}$ which have the same neighbors except that $\varepsilon_{1}$ intersects $\rho$ and $\varepsilon_{2}$ does not while $\varepsilon_{2}$ intersects $\lambda$ and $\varepsilon_{1}$ does not. Then $\varepsilon_{1}$ and $\varepsilon_{2}$ will be adjacent to every point in $P$. If $P$ is complete then

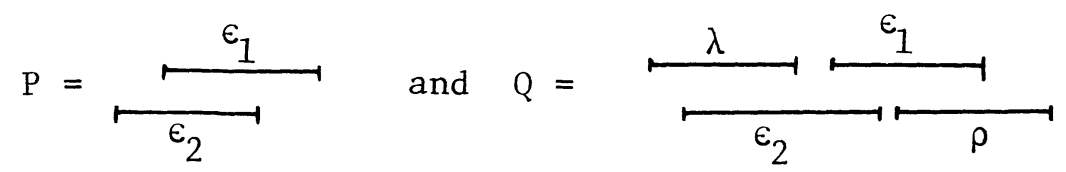

or
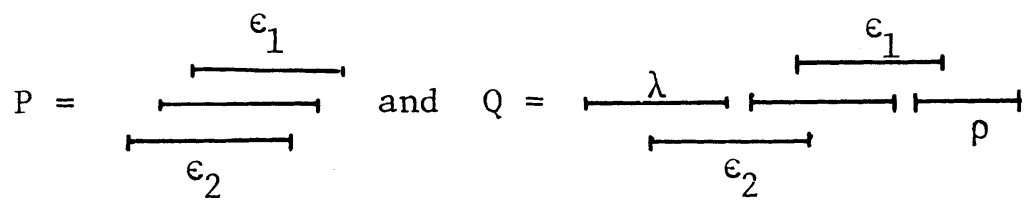

Suppose $P$ is not complete. Let $A$ be the set of points adjacent to every point in $P$. Then $B=V(P)-A$ is a buried subgraph of $P$ and so the graph induced by $(V(P)-A) \cup\left\{\varepsilon_{1}\right\}$ is a reduced, unit interval graph with a buried subgraph. By 
Lemma 4 we know this graph is $S_{n}$ for some $n$. So if $P$ is not complete we have either:
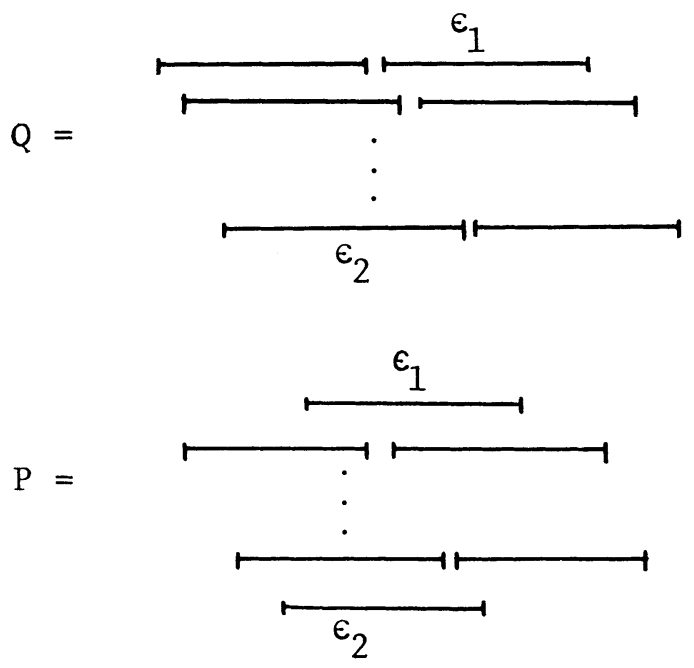

or
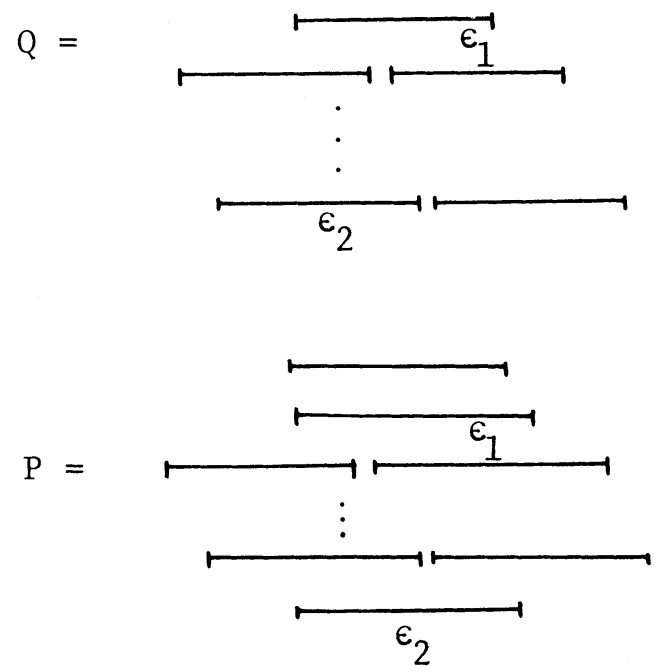

We can now establish recursions for the number of symmetric, reduced unit interval schemes. The predecessor of a symmetric, reduced, unit interval scheme $Q$ fails to be reduced only for the few schemes $Q$ given above. We will never form their predecessors; instead we will include these schemes $Q$ in our initial conditions. Let $e(r, s)$ denote the number of symmetric, reduced unit interval schemes with $r$ intervals and $s$ pairs of end-intervals (one on the left and one on the right). 
THEOREM 8. The numbers $e(r, s)$ satisfy the boundary conditions and recursion given by

(a) $e(1, s)=e(2, s)=0$ for all $s$.

(b) $e(r, 1)=0$ for all $r$.

(c) $e(r, s)=0$ if $s>|(r+1) / 2|$.

(d) $e(2 k-1, k)=e(2 k, k)=1$ for $k=2,3,4, \ldots$.

(e) $e(5,2)=1$.

(f) $e(r, s)=\sum_{i=s}^{\infty} e(r-2, i)+\sum_{i=s-1}^{\infty} e(r-4, i)$ for $r \geqslant 6$ and for $2 \leqslant s \leqslant$ $|(r+1) / 2|$.

\begin{tabular}{r|rrrrrrrr}
$r \backslash s$ & 1 & 2 & 3 & 4 & 5 & 6 & 7 & Total \\
\hline 1 & 0 & 0 & 0 & 0 & 0 & 0 & 0 & 0 \\
2 & 0 & 0 & 0 & 0 & 0 & 0 & 0 & 0 \\
3 & 0 & 1 & 0 & 0 & 0 & 0 & 0 & 1 \\
4 & 0 & 1 & 0 & 0 & 0 & 0 & 0 & 1 \\
5 & 0 & 2 & 1 & 0 & 0 & 0 & 0 & 3 \\
6 & 0 & 1 & 1 & 0 & 0 & 0 & 0 & 2 \\
7 & 0 & 4 & 2 & 1 & 0 & 0 & 0 & 7 \\
8 & 0 & 3 & 2 & 1 & 0 & 0 & 0 & 6 \\
9 & 0 & 10 & 6 & 2 & 1 & 0 & 0 & 19 \\
10 & 0 & 8 & 5 & 2 & 1 & 0 & 0 & 16 \\
11 & 0 & 26 & 16 & 6 & 2 & 1 & 0 & 51 \\
12 & 0 & 22 & 14 & 6 & 2 & 1 & 0 & 45 \\
13 & 0 & 70 & 44 & 18 & 6 & 2 & 1 & 141 \\
14 & 0 & 61 & 39 & 17 & 6 & 2 & 1 & 126
\end{tabular}

TABLE II. $e(r, s)$

Let $m_{r}$ denote the number of reduced, unit interval schemes with $r$ intervals and let $n_{r}$ denote the number of symmetric, reduced unit interval schemes with $r$ intervals. The recursions given in Theorems 7 and 8 allow us to compute $m_{r}$ and $n_{r}$ since $m_{1}=1$ and $m_{r}=\sum_{s=2}^{\infty} a(r, s)$ for $r>2$, and $n_{1}=1$ and $n_{r}=\sum_{s=2}^{\infty} e(r, s)$ for $r>2$. Define the generating functions $M(z)$ and $N(z)$ by $M(z)=\sum_{r=1}^{\infty} m_{r} z^{r}$ and $N(z)=\sum_{r=1}^{\infty} n_{r} z^{r}$. Let $P(z), U(z)$ and $C^{u}(z)$ be the ordinary generating functions for reduced unit interval graphs, unit interval graphs and connected unit interval graphs. Let $J^{u}(z)$ and $K^{u}(z)$ be the ordinary generating functions for identity unit interval graphs and connected identity unit interval graphs. Lastly, let $\Lambda(z)$ and $\Gamma(z)$ be the exponential generating functions for labelled unit interval graphs and connected labelled unit graphs.

Consider the generating function $N(z)$. We can write $N(z)=N_{e}\left(z^{2}\right)+z N_{0}\left(z^{2}\right)$ where

$$
N_{e}(z)=\sum_{k=1}^{\infty} \alpha_{k} z^{k} \text { and } N_{0}(z)=\sum_{k=1}^{\infty} \beta_{k} z^{k}
$$

satisfy $\alpha_{k}=n_{2 k}$ and $\beta_{k}=n_{2 k+1}$. We split the generating function $N(z)$ like that because a symmetric reduced unit interval scheme with an odd number of intervals 
has exactly one symmetric interval. When we build symmetric connected unit interval schemes from symmetric reduced unit interval schemes we will need to let single intervals be replaced by stacks of intervals. Pairs of opposite intervals must be replaced by stacks of the same height, but the single symmetric interval is opposite itself and so must be treated separately during this operation.

We can now produce all 7 of the generating functions $P(z), C^{u}(z), U(z), J^{u}(z)$, $K^{u}(z), \Gamma(z)$ and $\Lambda(z)$ from the known generating functions $M(z)$ and $N(z)$. The equations to do so are given in the following theorem.

THEOREM 9. The following generating function equations hold:

(c) $1+U(z)=\exp \left(\sum_{k=1}^{\infty} \frac{C^{u}\left(z^{k}\right)}{k}\right)$.

(d) $\quad K^{u}(z)=\frac{1}{2}(M(z)-N(z))$.

(e) $1+J^{u}(z)=\exp \left(\sum_{k=1}^{\infty} \frac{(-1)^{k-1}}{k} K^{u}\left(z^{k}\right)\right)$.

(f) $\quad \Gamma(z)=\frac{1}{2} M\left(e^{z}-1\right)$.

(g) $1+\Lambda(z)=\exp (\Gamma(z))$.

Proof. We have already observed that (a) is true and (c) follows from (b) by a standard application of Polya's Theorem (see [11, p. 90]). The proof of (b) is just like the proof of Theorem 3 part (2). It rests on the observation that all connected unit interval schemes can be obtained from the reduced unit interval schemes by the processs of replacing individual intervals with vertical stacks of one or more intervals, all having the same endpoints.

The proofs of (d) and (c) follow the proof of Theorem 5 and the proofs of (f) and (g) follow the proof of Theorem 4.

It is worth pointing out that $U(z)$ is obtained from $C^{u}(z)$ using equation (c) and that $C^{u}(z)$ is obtained from $M(z)$ and $N(z)$ using (b). Similarly $J^{u}(z)$ is obtained from $K^{u}(z)$ via (e) and $\Lambda(z)$ is obtained from $\Gamma(z)$ via (g). Tables of $P(z), C^{u}(z)$, $U(z), K^{u}(z), J^{u}(z), \Gamma(z)$ and $\Lambda(z)$ appear in $\$ 9$. An analysis of the asymptotic growth rate of the coefficients of these generating functions appears in the next section.

7. The asymptotic number of unit interval graphs. In this section we derive asymptotic estimates for the number of unit interval graphs, connected unit interval graphs, reduced unit interval graphs and identity unit interval graphs.

Recall the double sequence $a(r, s)$ defined in the previous section and the sequences $m_{r}$ and $n_{r}$. Form the generating function $A(y, z)=\sum_{r, s} a(r, s) z^{r} y^{s-2}$. Note that $y$ appears to the power $(s-2)$ in $A(y, z)$ and so $A(0, z)=\sum_{r=3}^{\infty} a(r, 2) z^{r}$. 
Also, for $r \geqslant 4$ we have

$$
\begin{aligned}
a(r, 2) & =\sum_{i=2}^{\infty} a(r-1, i)+\sum_{i=1}^{\infty} a(r-2, i) \\
& =\sum_{i=2}^{\infty} a(r-1, i)+\sum_{i=2}^{\infty} a(r-2, i)=m_{r-1}+m_{r-2} .
\end{aligned}
$$

From this we easily derive the following lemma.

LEMMA 8. $A(0, z)=z^{3}+\left(z+z^{2}\right) M(z)$.

Analytically, this equation is valid for all $z$ in the circle of convergence about 0 of the two series $M(z)$ and $A(0, z)$. One can obtain a simple expression for $A(0, z)$ from $A(y, z)$ by relating the two algebraically and then substituting

$$
y=\left(1-z-\sqrt{1-2 z-3 z^{2}}\right) / 2 z^{2} .
$$

This derivation, suggested by R. C. Read, allows us to deduce Theorem 10 formally. However the above substitution takes us outside the circle of convergence of $A(y, z)$ near $z=0$. So we consider a closely related series $B(y, z)$ obtained from $A(y, z)$ by a change of variables.

Definition 8. Define the double sequence $b(r, s), r, s \in \mathbf{Z}$, recursively as follows:

(a) $b(r, s)=0$ if $r \leqslant 2$,

(b) $b(r, s)=0$ if $s \leqslant 1$,

(c) $b(3,2)=1$,

(d) $b(r, s)=\sum_{j=0}^{\infty}(b(r-2 j, s+j-1)+b(r-2 j-1, s+j))$ for $r \geqslant 3, s \geqslant 2$ and $(r, s) \neq(3,2)$.

\begin{tabular}{r|rrrr}
$r \backslash s$ & 2 & 3 & 4 & 5 \\
\hline 1 & 0 & 0 & 0 & 0 \\
2 & 0 & 0 & 0 & 0 \\
3 & 1 & 1 & 1 & 1 \\
4 & 1 & 2 & 3 & 4 \\
5 & 2 & 5 & 9 & 14 \\
6 & 4 & 12 & 25 & 44 \\
& \multicolumn{3}{|c}{ TABLE III. $b(r, s)$}
\end{tabular}

LEMMA 9. $b(r, s)=a(r+2 s-4, s)$ for $r \geqslant 1$ and $s \geqslant 1$.

Proof. Consider $r=1$ or 2 . Then for all $s \geqslant 1$ we have

$$
s>\left[\frac{\frac{1}{2}(r+2 s-4)+1}{2}\right]
$$

and so $a(r+2 s-4, s)=0=b(r, s)$.

For $r \geqslant 3$ we proceed by induction on the number $r+2 s-4$. If $r+2 s-4=3$ we must have $s=2$ and $b(3,2)=a(3,2)=1$. Consider $r+2 s-4>3$. We know

$$
b(r, s)=\sum_{j=0}^{\infty}(b(r-2 j, s+j-1)+b(r-2 j-1, s+j)) .
$$


Note that $(r-2 j)+2(s+j-1)-4=r+2 s-6<r+2 s-4$, and that $(r-$ $2 j-1)+2(s+j)-4=r+2 s-5<r+2 s-4$. By our induction hypothesis,

$$
\begin{aligned}
b(r, s) & =\sum_{j=0}^{\infty}(a(r+2 s-6, s+j-1)+a(r+2 s-5, s+j)) \\
& =\sum_{i=s-1}^{\infty} a((r+2 s-4)-2, i)+\sum_{i=s}^{\infty} a((r+2 s-4)-1, i) \\
& =a(r+2 s-4, s) .
\end{aligned}
$$

Note that $b(r, 2)=a(r, 2)$. Define the generating function $B(y, z)=$ $\Sigma_{r, s} b(r, s) z^{r} y^{s-2}$. Using Lemma 8, we have

$$
B(0, z)=z^{3}+\left(z+z^{2}\right) M(z) .
$$

We next need a technical lemma which assures us that $B(y, z)$ converges in the disk about the origin in $\mathbf{C} \times \mathbf{C}$ given by $|y|<1 / 16$ and $|z|<1 / 16$.

LEMMA 10. For all $r$ and $s$ we have

$$
b(r, s) 16^{-r} 16^{-s} \leqslant 2^{-r} 4^{-s} .
$$

Proof. This is obvious whenever $b(r, s)=0$. For nonzero values of $b(r, s)$ we proceed by induction on $r+2 s-4$. For $r+2 s-4=3$ we have $s=2$ and $r=3$ and $b(3,2) 16^{-3} 16^{-2}=16^{-5} \leqslant 2^{-3} 4^{-2}$. Consider $r+2 s-4>3$.

$$
\begin{aligned}
b(r, s) 16^{-r} 16^{-s}= & \sum_{j=0}^{\infty}\left(b(r-2 j, s+j-1) 16^{-r} 16^{-s}\right) \\
& +\sum_{j=0}^{\infty}\left(b(r-2 j-1, s+j) 16^{-r} 16^{-s}\right)
\end{aligned}
$$

so

$$
\begin{aligned}
b(r, s) 16^{-r} 16^{-s}= & \sum_{j=0}^{\infty} 16^{-(1+j)}\left(b(r-2 j, s+j-1) 16^{-(r+2 j)} 16^{-(s+j-1)}\right) \\
& +\sum_{j=0}^{\infty} 16^{-(1+j)}\left(b(r-2 j-1, s+j) 16^{-(r-2 j-1)} 16^{-(s+j)}\right) .
\end{aligned}
$$

By our induction hypothesis we have

$$
\begin{aligned}
b(r, s) 16^{-r} 16^{-s} \leqslant & \sum_{j=0}^{\infty} 16^{-(1+j)} 2^{-(r-2 j)} 4^{-(s+j-1)} \\
& +\sum_{j=0}^{\infty} 16^{-(1+j)} 2^{-(r-2 j-1)} 4^{-(s+j)} .
\end{aligned}
$$

Simplifying the sum on the right we have

$$
b(r, s) 16^{-r} 16^{-s} \leqslant 2^{-r} 4^{-s}\left(\frac{1}{4} \sum_{j=0}^{\infty} 16^{-j}+\frac{1}{8} \sum_{j=0}^{\infty} 16^{-j}\right)
$$

which completes the proof of this lemma. 
THEOREM 10. The power series $B(y, z)$ has a positive radius of convergence, converging for all $y$ and $z$ with $|y| \leqslant 1 / 16$ and $|z| \leqslant 1 / 16$.

Proof. As $B(y, z)$ has nonnegative real coefficients, it suffices to show that the doubly indexed sequence $\sigma(a, b)$ converges in the Pringsheim sense where

$$
\sigma(a, b)=\sum_{r \leqslant a} \sum_{s \leqslant b} b(r, s) 16^{-r} 16^{-s} .
$$

This follows immediately from the estimate given in Lemma 10.

We now use $B(y, z)$ to compute a simple algebraic expression for $B(0, z)$. From the equations in Definition 8, we have for all $r \geqslant 4$,

$$
\begin{aligned}
b(r, s)= & \sum_{j=0}^{\infty}(b(r-2 j, s+j-1)+b(r-2 j-1, s+j)) \\
= & b(r, s-1)+b(r-1, s) \\
& +\sum_{j=1}^{\infty}(b(r-2 j, s+j-1)+b(r-2 j-1, s+j)) .
\end{aligned}
$$

Letting $l=j-1$ we have

$$
\begin{aligned}
b(r, s)= & b(r, s-1)+b(r-1, s) \\
& +\sum_{l=0}^{\infty}(b((r-2)-2 l,(s+1)+l-1) \\
& +b((r-2)-2 l-1,(s+1)+l))
\end{aligned}
$$

and so

$$
b(r, s)=b(r, s-1)+b(r-1, s)+b(r-2, s+1) .
$$

Summing (7.2) over all $s \geqslant 2$ and multiplying each equation by $y^{s-1}$ we obtain

$$
\begin{aligned}
y \sum_{s=2}^{\infty} b(r, s) y^{s-2}= & y^{2} \sum_{s=2}^{\infty} b(r, s-1) y^{s-3} \\
& +y \sum_{s=2}^{\infty} b(r-1, s) y^{s-2}+\sum_{s=2}^{\infty} b(r-2, s+1) y^{s-1} .
\end{aligned}
$$

Let $B_{r}(y)=\sum_{s=2}^{\infty} b(r, s) y^{s-2}$; note that $B(y, z)=\sum_{r=3}^{\infty} B_{r}(y) z^{r}$. Rewriting (7.3) we obtain for $r>3$

$$
\begin{aligned}
y B_{r}(y) z^{r}= & y^{2} B_{r}(y) z^{r}+y z B_{r-1}(y) z^{r-1} \\
& +z^{2}\left(B_{r-2}(y) z^{r-2}-b(r-2,2) z^{r-2}\right) .
\end{aligned}
$$

Also

$$
y B_{3}(y) z^{3}=y z^{3} /(1-y)=y z^{3}+y^{2} B_{3}(y) z^{3} .
$$

Noting that $B_{2}(y)=B_{1}(y)=0$ we can rewrite (7.5) as

(7.6) $y B_{3}(y) z^{3}=y x z^{3}+y^{2} B_{3}(y) z^{3}+y z B_{2}(y) z^{2}+z^{2}\left(B_{1}(y) z-b(1,2) z\right)$.

Summing (7.6) together with (7.4) for $r \geqslant 4$ gives $y B(y, z)=y^{2} B(y, z)+$ $y z B(y, z)+z^{2} B(y, z)+z^{3} y-z^{2} B(0, z)$ which can be rewritten as

$$
\left(\left(y^{2}-y\right)+y z+z^{2}\right) B(y, z)=-z^{3} y+z^{2} B(0, z) \text {. }
$$


We now evaluate (7.7) along the curve

$$
y=\frac{1}{2}\left(1-z-\sqrt{(z-1)^{2}-4 z^{2}}\right) .
$$

Note that as $z$ approaches 0 , the variable $y$ approaches 0 continuously; hence we may choose $\beta$, with $0<\beta<1 / 16$, such that if $|z|<\beta$ then $|y|<1 / 16$. For such pairs $(y, z)$ the series $B(y, z)$ converges to a finite number by Theorem 10 , and so if $y$ also satisfies (7.8) the left-hand side of (7.7) is 0 . We obtain

$$
B(0, z)=\frac{1}{2}\left(1-z-\sqrt{1-2 z-3 z^{2}}\right)
$$

for all $z$ with $|z|<\beta$.

THEOREM 11. $M(z)=\frac{1}{2}(1-2 z)-\frac{1}{2} \sqrt{(1-3 z) /(1+z)}$.

Proof. Combining equation (7.1) with (7.9) we have

$$
\left(z+z^{2}\right) M(z)=-z^{3}+z\left(\frac{1-z-\sqrt{(1-3 z)(1+z)}}{2}\right)
$$

so

$$
M(z)=-\frac{z^{2}}{1+z}+\frac{1-z}{2(1+z)}-\frac{1}{2} \sqrt{\frac{(1-3 z)}{1+z}} .
$$

Hence $M(z)=\left(1-z-2 z^{2}\right) / 2(1+z)-\frac{1}{2} \sqrt{(1-3 z) /(1+z)}$. Theorem 11 tells us that $M(z)$ has a square-root type singularity at $1 / 3$. With two exceptions the coefficient of $z^{r}$ in the Taylor expansion of $M(z)$ about the origin is the same as the coefficient of $z^{r}$ in the Taylor expansion of $-\frac{1}{2} \sqrt{(1-3 z) /(1+z)}$. We can obtain a rough asymptotic estimate for $m_{r}$ at this point.

COROllary 11.1.

$$
m_{r} \sim\left(\frac{\sqrt{3}}{8 \sqrt{\pi}}\right) \frac{3^{r}}{r^{3 / 2}}
$$

Proof. Write $M(z)=\alpha(z) \beta(z)$ where $\alpha(z)=(1+z)^{-1 / 2}$ and $\beta(z)=$ $-\frac{1}{2}(1-3 z)^{1 / 2} \cdot \alpha(z)$ and $\beta(z)$ have radii of convergence 1 and $1 / 3$ respectively and $\beta_{r} / \beta_{r-1}$ approaches the value 3 as $r \rightarrow \infty$. Apply Theorem 2 of Bender (see [1, p. 496]); we have $m_{r} \sim \alpha(1 / 3) \beta_{r} \sim(\sqrt{3} / 2) \beta_{r}$. Using the Binomial Theorem we have

$$
\beta_{r}=\left(\frac{-1}{2}\right)(-3)^{r}\left(\begin{array}{c}
\frac{1}{2} \\
r
\end{array}\right)
$$

so

$$
\beta_{r}=\left(\frac{3}{4}\right)^{r}\left(\begin{array}{c}
2 r-2 \\
r-1
\end{array}\right) \frac{1}{r} .
$$

By Stirling's Formula we have

$$
\beta_{r} \sim \frac{1}{r}\left(\frac{3}{4}\right)^{r} \frac{1}{\sqrt{\pi}} \frac{4^{r-1}}{\sqrt{r-1}} .
$$

Substituting this estimate for $\beta_{r}$ completes the proof. 
The following table gives some idea of the rate of convergence of this estimate for $m_{r}$.

\begin{tabular}{r|r|r|r}
$r$ & $e_{r}=\left(\frac{\sqrt{3}}{8 \sqrt{\pi}}\right) \frac{3^{r}}{r^{3 / 2}}$ & $m_{r}$ & $m_{r} / e_{r}$ \\
\hline 3 & .63471 & 1 & 1.57551 \\
4 & 1.23677 & 1 & .80855 \\
5 & 2.65489 & 3 & 1.12998 \\
6 & 6.05893 & 6 & .99027 \\
7 & 14.42438 & 15 & 1.03990 \\
8 & 35.41854 & 36 & 1.01641 \\
9 & 89.04780 & 91 & 1.02192 \\
10 & 228.09106 & 232 & 1.01713 \\
15 & 30170.162 & 30537 & 1.01215 \\
25 & $8.2794 \times 10^{8}$ & 834086421 & 1.00738
\end{tabular}

TABLE IV. Estimates for $m_{r}$

We next perform similar analyses of the series $N_{e}(z)$ and $N_{0}(z)$ beginning with $N_{0}(z)$. Recall the doubly indexed sequence $e(r, s)$ from $\S 6$. The subsequence consisting of those terms with $r$ even satisfies the recurrence:

(a) $e(2, s)=0$ for all $s$.

(b) $e(2 k, 1)=0$ for all $k$.

(c) $e(2 k, s)=0$ if $s>k$.

(d) $e(2 k, k)=1$ for $k=2,3, \ldots$.

(e) $e(2 k, s)=\sum_{i=s}^{\infty} e(2 k-z, i)+\sum_{i=s-1}^{\infty} e(2 k-4, i)$ for $k \geqslant 3$ and $2 \leqslant s<k$. The $k$ th coefficient of $N_{e}(z)$ is given by $\sum_{s=2}^{\infty} e(2 k, s)$.

For convenience of notation we will again name a series $b(r, s)$. The derivation here is different than in the case of $M(z)$ but the ideas are much the same. Hence fewer details are included.

Define $b(r, s)$ by

\begin{tabular}{c|rrrr}
$b(r, s)=e((2 r+2 s-4), s)$ for all $r, s$. \\
$r \backslash s$ & 2 & 3 & 4 & 5 \\
\hline 4 & 1 & 0 & 0 & 0 \\
6 & 1 & 1 & 0 & 0 \\
8 & 3 & 2 & 1 & 0 \\
10 & 8 & 5 & 2 & 1 \\
& & & &
\end{tabular}




\begin{tabular}{c|rrrr}
$r \backslash^{s}$ & 2 & 3 & 4 & 5 \\
\hline 2 & 1 & 1 & 1 & 1 \\
3 & 1 & 2 & 2 & 2 \\
4 & 3 & 5 & 6 & 6 \\
5 & 8 & 14 & 17 & 18 \\
& & &
\end{tabular}

Using the recursion for $e(r, s)$ set out above it is easy to check that for $r \geqslant 3$ we have

$$
b(r, s)=\sum_{l=0}^{\infty}(b(r-l-1, s+l)+b(r-l-1, s+l-1)) .
$$

So

$$
\begin{aligned}
b(r, s)= & b(r-1, s)+b(r-1, s-1) \\
& +\sum_{j=0}^{\infty}(b((r-1)-j-1,(s+1)+j) \\
& +b(((r-1)-j-1,(s+1)+j-1))) .
\end{aligned}
$$

For $r \geqslant 4$ (hence $(r-1) \geqslant 3$ ) the two equations above combine to give

$$
b(r, s)=b(r-1, s)+b(r-1,3-1)+b(r-1, s+1) .
$$

As before, let $B(y, z)=\Sigma_{r, s} b(r, s) z^{r} y^{s-2}$. Using (7.10) it can be shown inductively that $b(r, s)=23^{r-3}$ for $r \geqslant 3$ and $s \geqslant r$. Thus $B(y, z)$ converges for all $y, z$ with $|z|<1$ and $|y|<1 / 3$. Also it is easy to check that $z^{2}+\left(z+z^{2}\right) N_{e}(z)=B(0, z)$ or equivalently

$$
N_{e}(z)=-z /(1+z)+B(0, z) /\left(z+z^{2}\right) .
$$

From (7.10) one can derive

$$
\begin{aligned}
y B(y, z)= & y z B(y, z)+y^{2} z B(y, z)+z B(y, z) \\
& -z B(0, z)-\frac{y z^{3}}{1-y}+\frac{y z^{2}}{1-y} .
\end{aligned}
$$

This can be rewritten as

$$
B(y, z)\left(y^{2} z+y(z-1)+z\right)=z B(0, z)+y\left(z^{3}-z^{2}\right) /(1-y) .
$$

Evaluate (7.13) along the curve

$$
y=\frac{(1-z)-\sqrt{(z-1)^{2}-4 z^{2}}}{2 z}=\frac{(1-z)-\sqrt{1-2 z-3 z^{2}}}{2 z} .
$$

Using L'Hôpital's rule one finds that $y$ goes to 0 as $z$ goes to 0 ; hence for small $z$ we have $|y|<1 / 3$ and so $B(y, z)$ converges. Now some simple calculations show that

$$
\frac{y}{1-y}=\frac{1}{2}\left(-1+\sqrt{\frac{1+z}{1-3 z}}\right)
$$


and so for $z$ near 0 we have

$$
z B(0, z)=\left(z-z^{3}\right)\left(-\frac{1}{2}+\frac{1}{2} \sqrt{(1+z) /(1-3 z)}\right)
$$

or $B(0, z)=z(1-z)\left(-\frac{1}{2}+\frac{1}{2} \sqrt{(1+z) /(1-3 z)}\right)$. Hence

$$
N_{e}(z)=-\frac{z}{1+z}+\left(\frac{1-z}{1+z}\right)\left(-\frac{1}{2}+\frac{1}{2} \sqrt{\frac{1+z}{1-3 z}}\right) .
$$

Equation (7.14) can be further simplified to

$$
N_{e}(z)=-\frac{1}{2}+\frac{1}{2}\left(\frac{(1-z)}{\sqrt{(1+z)(1-3 z)}}\right) .
$$

We see that $N_{e}(z)$ has radius of convergence $1 / 3$ and so $N_{e}\left(z^{2}\right)$ has radius of convergence $1 / \sqrt{3}$.

The analysis of $N_{0}(z)$ is almost identical. The equations differ slightly due to the boundary condition $e(5,2)=2$. The final equation reads as follows:

$$
N_{0}(z)=-1+\left(1-2 z-3 z^{2}\right)^{-1 / 2} \text {. }
$$

We see that $N_{0}(z)$ has radius of convergence $1 / 3$ and so $z N_{0}\left(z^{2}\right)$ has radius of convergence $1 / \sqrt{3}$.

Using (7.15) we have that the $r$ th coefficient of $N_{e}(z)$ is asymptotic to $3^{r} / 2 \sqrt{3 \pi r}$ and using (7.16) we have that the $r$ th coefficient of $N_{0}(z)$ is asymptotic to $3^{r+1 / 2} / 2 \sqrt{\pi r}$

We can combine this in the following theorem.

THEOREM 12. Let $p_{r}, c_{r}^{u}$ and $u_{r}$ denote the number of reduced unit interval graphs, connected unit interval graphs and unit interval graphs on $r$ points. Then

(A)

$$
P(z)=\frac{1}{4} \frac{\left(1+2 z-z^{2}\right)}{\left(1+z^{2}\right)^{1 / 2}\left(1-3 z^{2}\right)^{1 / 2}}-\frac{1}{4} \frac{(1-3 z)^{1 / 2}}{(1+z)^{1 / 2}} \text { and }
$$

(B)

$$
\begin{gathered}
P_{r} \sim\left(\frac{\sqrt{3}}{16 \sqrt{\pi}}\right) \frac{3^{r}}{r^{3 / 2}} . \\
C^{u}(z)=\frac{\left(z+z^{2}\right)}{2\left(1-4 z^{2}\right)^{1 / 2}}+\frac{\left(1-2 z^{2}\right)}{4\left(1-4 z^{2}\right)^{1 / 2}}-\frac{1}{4}(1-4 z)^{1 / 2}, \\
c_{r}=\left(\frac{1}{8 \sqrt{\pi}}\right)\left(\frac{4^{r}}{r^{3 / 2}}\right)\left(1-\frac{1}{8 r}+O\left(\frac{1}{r^{2}}\right)\right) .
\end{gathered}
$$

(C) $c_{r} / u_{r} \sim K$ where $K$ is a constant $(K=.626578 \ldots) . K$ is the value $\exp (-\sqrt{3} / 4) \exp \left(-\sum_{l=2}^{\infty} C^{u}\left(4^{-l}\right) / l\right)$.

$$
u_{r} \sim\left(\frac{1}{8 K \sqrt{\pi}}\right)\left(\frac{4^{r}}{e^{3 / 2}}\right)
$$

Proof. To prove (A) simply combine Theorem 9(a) with Theorem 11, (7.15), (7.16) and Corollary 11.1. 
To prove the functional equation given in (B) combine Theorem 9(b) with Theorem 11, (7.15) and (7.16).

We-next prove the estimate given in (B). Let

$$
f(z)=\frac{\left(z+z^{2}\right)}{2\left(1-4 z^{2}\right)^{1 / 2}}+\frac{\left(1-2 z^{2}\right)}{4\left(1-4 z^{2}\right)^{1 / 2}}=\sum_{r=0}^{\infty} f_{r} z^{r} .
$$

The $n$th coefficient of $(1-4 z)^{-1 / 2}$ is

$$
(-r)^{n}\left(\begin{array}{c}
-1 / 2 \\
n
\end{array}\right)
$$

which is equal to $\left(2^{3 n} / \sqrt{\pi n}\right)\left(1+O\left(\frac{1}{n}\right)\right)$ by Stirling's formula. Hence the $r$ th coefficient of $f(z)$ satisfies

$$
f_{r} \sim K_{1} 2^{3 r / 2} / r^{1 / 2}
$$

where $K_{1}$ is a constant. Using the Binomial Theorem and Stirling's formula again we find that the $r$ th coefficient of $(1-4 z)^{1 / 2} / 4$ has size

$$
\frac{1}{8 \sqrt{\pi}}\left(\frac{4^{r}}{r^{3 / 2}}\right)\left(1-\frac{1}{8 r}+O\left(\frac{1}{r^{2}}\right)\right) .
$$

The estimate given in (B) follows immediately.

We now prove (C). By Theorem 9(c) we have

$$
1+U(z)=\exp \left(\sum_{l=1}^{\infty} \frac{C^{u}\left(z^{l}\right)}{l}\right) .
$$

Write $1+U(z)=f(z) \exp \left(\sum_{l=1}^{\infty}\left(h\left(z^{l}\right) / l\right)\right)$ where

$$
h(z)=\frac{\left(z+z^{2}\right)}{2\left(1-4 z^{2}\right)^{1 / 2}}+\frac{\left(1-2 z^{2}\right)}{4\left(1-4 z^{2}\right)^{1 / 2}}-\frac{1}{4},
$$

and

$$
f(z)=\exp \left(\sum_{l=1}^{\infty} \frac{1-\left(1-4 z^{l}\right)^{1 / 2}}{4 l}\right) .
$$

The function $f(z)$ has radius of convergence $1 / 4$ and the function $h(z)$ has radius of convergence $1 / 2$. It follows that

$$
u_{r} \sim f_{r} \exp \left(\sum_{l=1}^{\infty} \frac{h\left(4^{-l}\right)}{l}\right) .
$$

We now establish the size of $f_{r}$.

Let $X(z)=\sum_{r=0}^{\infty} x_{r} z^{r}$ denote the function $\frac{1}{4}-(1-4 z)^{1 / 2} / 4$. Then

$$
f(z)=\exp \left(\sum_{l=1}^{\infty} \frac{X\left(z^{l}\right)}{l}\right)=\exp (X(z)) \exp \left(\sum_{l=2}^{\infty} \frac{X\left(z^{l}\right)}{l}\right) .
$$

Let $y_{r}$ denote the $r$ th coefficient of $\sum_{l=2}^{\infty}\left(X\left(z^{l}\right) / l\right)$. Then

$$
y_{r}=\sum_{\substack{d \mid r \\ d>1}} \frac{x_{r / d}}{d} \leqslant \frac{r}{2} X_{[r / 2]} \leqslant 2^{r} .
$$


The last inequality holds for large $r$ since $X_{r} \sim(1 / 8 \sqrt{\pi})\left(4^{r} / r^{2 / 3}\right)$ (see part (B)). So the series $\exp \left(\sum_{l=2}^{\infty}\left(X\left(z^{l}\right) / l\right)\right)$ has radius of convergence $1 / 2$ or greater. Hence

$$
f_{r} \sim \exp (1 / 4) \exp \left(\sum_{l=2}^{\infty} \frac{X\left(4^{-l}\right)}{l}\right) z_{r}
$$

where $z_{r}$ is the $r$ th coefficient of $\exp \left(-(1-4 z)^{1 / 2} / 4\right)$. Note that $X(z)+h(z)=$ $C^{u}(z)$. Combining (7.18) with (7.19) yields

$$
u_{r} \sim \exp \left(\frac{1}{4}+h\left(\frac{1}{4}\right)+\sum_{l=2}^{\infty} \frac{C^{u}\left(4^{-l}\right)}{l}\right) z_{r} .
$$

As $h(1 / 4)=(\sqrt{3}-1) / 4$ we have

$$
u_{r} \sim \exp \left(\frac{\sqrt{3}}{4}\right) \exp \left(\sum_{l=2}^{\infty} \frac{C^{u}\left(4^{-l}\right)}{l}\right) z_{r} .
$$

It remains to show that $z_{r} \sim c_{r}$.

We write

$$
\exp \left(-\frac{1}{4}(1-4 z)^{1 / 2}\right)=\cosh \left(-\frac{1}{4}(1-4 z)^{1 / 2}\right)+\sinh \left(-\frac{1}{4}(1-4 z)^{1 / 2}\right) .
$$

Since $\cosh (u)=\cosh (-u)$ we see that $\cosh \left(-\frac{1}{4}(1-4 z)^{1 / 2}\right)$ is entire; hence the size of its coefficients is negligible for large $r$. The function

$$
-4(1-4 z)^{-1 / 2} \sinh \left(-\frac{1}{4}(1-4 z)^{1 / 2}\right)
$$

is analytic at $\frac{1}{4}$ and has the Taylor expansion

$$
1+\sum_{l=1}^{\infty} \frac{(1-4 z)^{l}}{16^{l}(2 l+1) !}
$$

The root test shows that this function is entire; note that it has value 1 at $\frac{1}{4}$. So, within this disk $|z|<\frac{1}{4}$ we have the functional equation

$$
\sinh \left(-\frac{1}{4}(1-4 z)^{1 / 2}\right)=-\frac{(1-4 z)^{1 / 2}}{4}\left(1+\sum_{l=1}^{\infty} \frac{(1-4 z)^{l}}{16^{l}(2 l+1) !}\right)
$$

from which it follows that $z_{r}$ is asymptotic to the $r$ th coefficient of $-(1-4 z)^{1 / 2} / 4$ which is asymptotic to $c_{r}^{u}$ by part (B). This completes the proof of part (c).

\begin{tabular}{r|c|c|c}
$r$ & $c_{r}^{u}$ & $\frac{1}{8 \sqrt{\pi}} \frac{4^{r}}{r^{3 / 2}}\left(1-\frac{1}{8 r}\right)$ & $c_{r}^{u} / u_{r}$ \\
\hline 1 & 1 & .246832 & 1 \\
5 & 10 & 6.297738 & .47619 \\
10 & 2494 & 2309.254 & .55397 \\
25 & $6.449534 \times 10^{\prime \prime}$ & $6.320448 \times 10^{\prime \prime}$ & .60700 \\
50 & $2.547761 \times 10^{26}$ & $2.522275 \times 10^{26}$ & .61568 \\
100 & $1.137544 \times 10^{56}$ & $1.131855 \times 10^{56}$ & .61952
\end{tabular}

TABLE V. Estimates for $c_{r}^{u}$ 
Lastly it should be mentioned that Professor Joel Cohen of Rockefeller University has results on the asymptotic probability that a random graph is a unit interval graph (see Cohen [3]). It seems very difficult to relate his results to those given in this section.

8. Conclusion. Several generalizations of interval graphs suggest open enumeration problems. Many people have studied interval representations in which each vertex corresponds to a set of intervals instead of just a single interval (see Griggs [7], Griggs and West [8], Harary and Kabell [10], and Harary and Trotter [12]). Such a graph is called a multiple interval graph. One can ask how many graphs have a multiple interval representation in which each vertex is allowed to correspond to a set of size $t$ or less. Another problem is to count higher dimensional interval graphs, where the vertices correspond to boxes in $k$-dimensional real space with two vertices adjacent if their corresponding boxes intersect.

As mentioned earlier, circular arc graphs can be counted using essentially the techniques of this paper, though to count circular arc graphs, interval graphs or even unit interval graphs by points and lines is an open problem. The techniques of this paper do not seem at all amenable to adding lines as a parameter.

Theorem 1 permits one to classify the abstract groups which are realized as automorphism groups of interval graphs and of unit interval graphs. Those which can be realized as automorphism groups of interval graphs are exactly those which can be realized as automorphism groups of trees. An interesting problem is to find for each interval graph $G$ a tree with $\Gamma(G) \cong \Gamma(T)$.

One can also ask for the asymptotic number of interval graphs. Cohen, Komlós and Mueller [4] have obtained probabilistic information but not an asymptotic expansion for the number of interval graphs on $r$ points. At present it is not even known whether $I(y)$ has a positive radius of convergence.

9. Numerical results. The algorithms developed in this paper to count interval graphs were programmed by Mr. Albert Nymeyer of the University of Newcastle, New South Wales, Australia. The programs were run on a PDP 11-45 and made use of multiple precision integer routines developed by Mr. Nymeyer and Dr. Paul Butler, also of the University of Newcastle. Mr. Nymeyer did this work in the course of research on a project directed by $\mathrm{R}$. W. Robinson and sponsored by the Australian Research Grants Committee. Thanks are due for his patient and competent programming.

Below is a list of what numbers are available. The line "Interval Graphs 30" means that the number of interval graphs has been computed through order 30 . Following this list are tables of some of these numbers.

1. Unlabelled Interval Graphs $\quad 30$

2. Unlabelled Connected Interval Graphs 30

3. Labelled Interval Graphs 30

4. Labelled Connected Interval Graphs 30 
5. Unlabelled Identity Interval Graphs 30

6. Unlabelled Connected Identity Interval Graphs 30

7. Unlabelled Unit Interval Graphs 190

8. Unlabelled Connected Unit Interval Graphs 190

9. Unlabelled Identity Unit Interval Graphs 190

10. Unlabelled Connected Identity Unit Interval Graphs 190

11. Labelled Unit Interval Graphs $\quad 100$

12. Labelled Connected Unit Interval Graphs $\quad 100$

\begin{tabular}{ll}
$n$ & $i_{n}$ \\
\hline 1 & 1 \\
2 & 2 \\
3 & 4 \\
5 & 10 \\
6 & 92 \\
7 & 369 \\
8 & 1,807 \\
9 & 10,344 \\
10 & 67,659 \\
11 & 491,347 \\
12 & $3,894,446$ \\
13 & $33,278,992$ \\
14 & $304,256,984$ \\
15 & $2,960,093,835$ \\
16 & $30,523,315,419$ \\
17 & $332,524,557,107$ \\
18 & $3,816,805,831,381$ \\
19 & $46,048,851,321,131$ \\
20 & $582,691,924,941,142$ \\
21 & $7,717,878,059,859,874$ \\
22 & $106,806,430,860,694,984$ \\
23 & $1,541,683,193,805,924,288$ \\
24 & $23,173,865,491,070,682,522$ \\
25 & $362,209,519,663,956,668,230$ \\
26 & $5,878,587,479,718,492,203,687$ \\
27 & $98,939,470,892,276,743,701,430$ \\
28 & $1,724,711,502,252,489,780,581,806$ \\
29 & $31,103,753,931,305,402,201,986,962$ \\
30 & $579,679,210,832,345,934,289,138,787$ \\
&
\end{tabular}

TABLE VII. Interval graphs 


\begin{tabular}{ll}
$n$ & $c_{n}$ \\
\hline 1 & 1 \\
2 & 1 \\
3 & 2 \\
4 & 5 \\
5 & 15 \\
10 & 54,962 \\
15 & $2,616,100,423$ \\
20 & $532,353,636,671,777$ \\
25 & $337,356,131,293,123,596,221$ \\
30 & $546,729,917,275,302,463,301,546,477$
\end{tabular}

TABLE VIII. Connected interval graphs

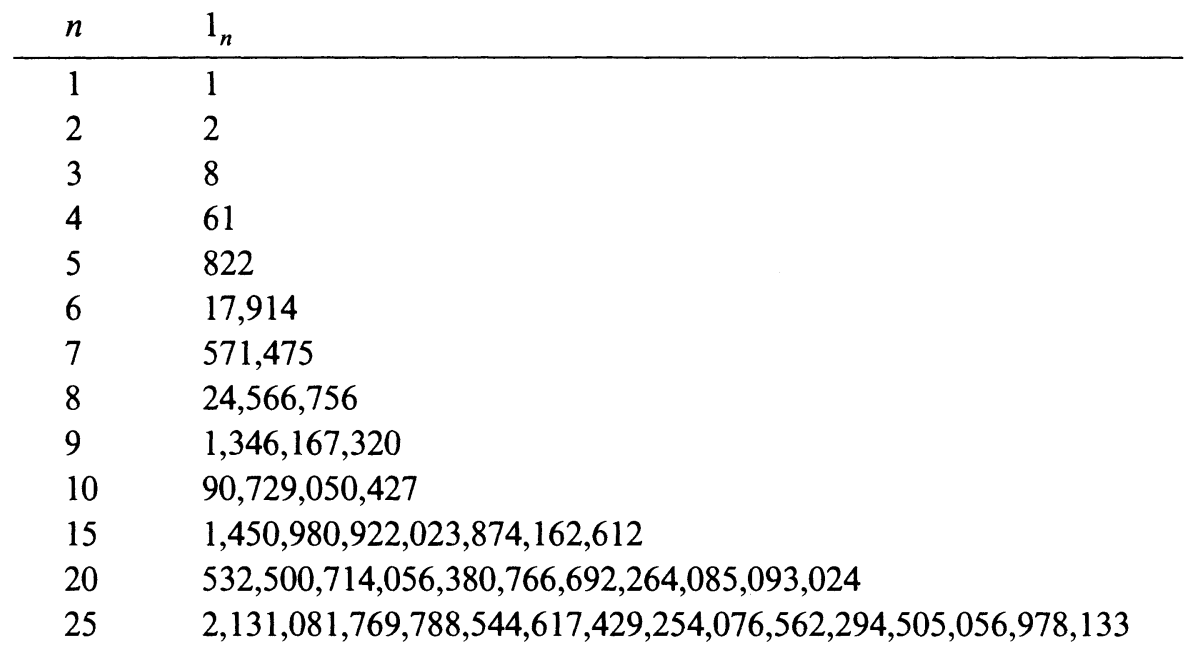

TABLE IX. Labelled interval graphs

\begin{tabular}{ll}
$n$ & $c_{n}^{L}$ \\
\hline 1 & 1 \\
2 & 1 \\
3 & 4 \\
5 & 541 \\
10 & $77,048,430,033$ \\
15 & $1,300,613,343,254,300,891,316$ \\
20 & $490,332,535,341,265,782,960,697,784,166,741$ \\
25 & $1,994,765,784,437,054,370,880,783,223,516,341,080,708,388,161$
\end{tabular}

TABLE X. Labelled connected interval graphs 


\begin{tabular}{ll}
$n$ & $j_{n}$ \\
\hline 1 & 1 \\
2 & 0 \\
3 & 0 \\
4 & 0 \\
5 & 0 \\
6 & 4 \\
7 & 31 \\
8 & 199 \\
9 & 1,313 \\
10 & 9,158 \\
15 & $416,581,477$ \\
20 & $82,900,013,695,783$ \\
25 & $52,634,658,108,249,193,680$ \\
30 & $86,079,203,001,046,903,539,459,019$
\end{tabular}

TABLE XI. Identity interval graphs

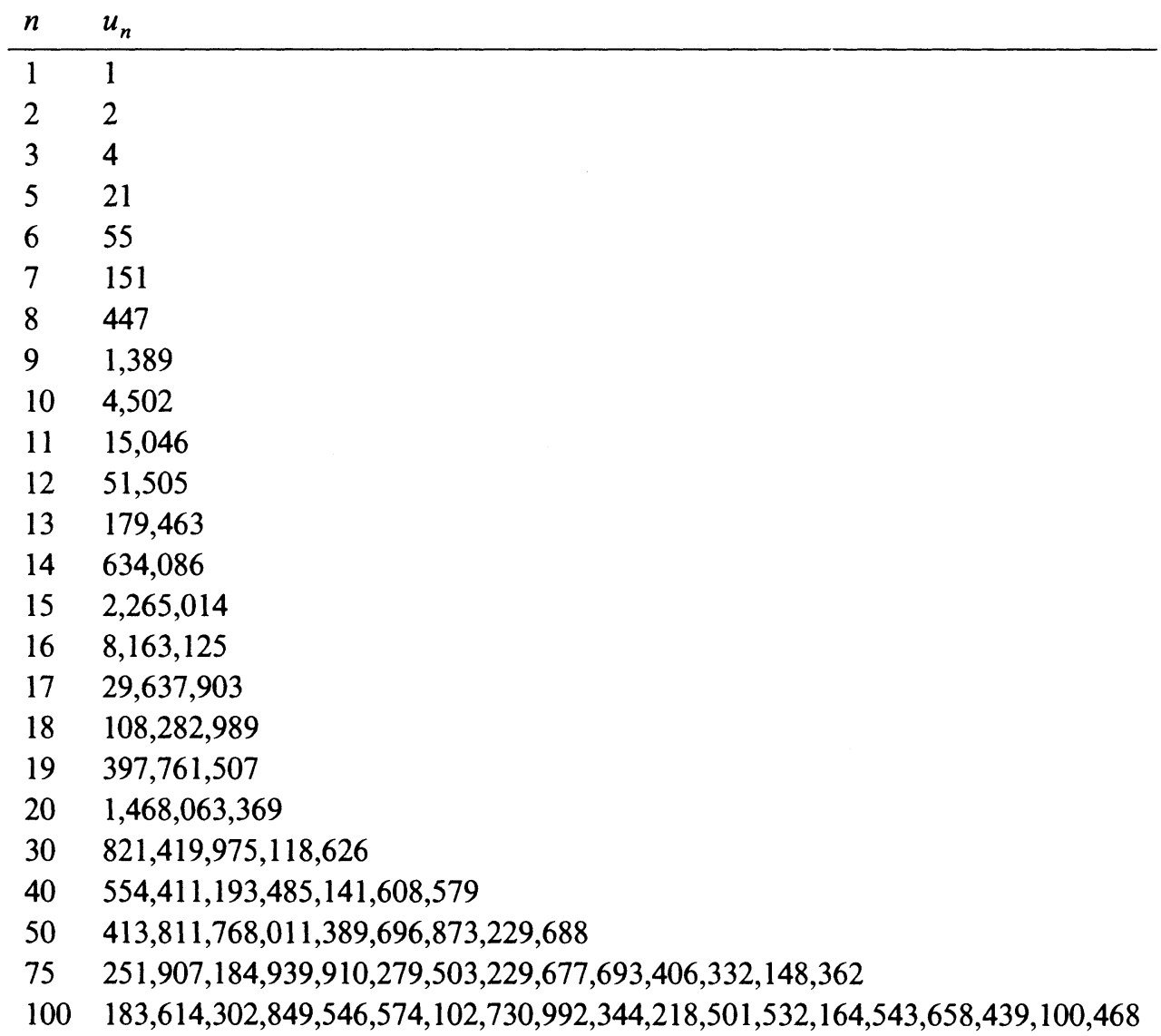

TABLE XII. Unit interval graphs 


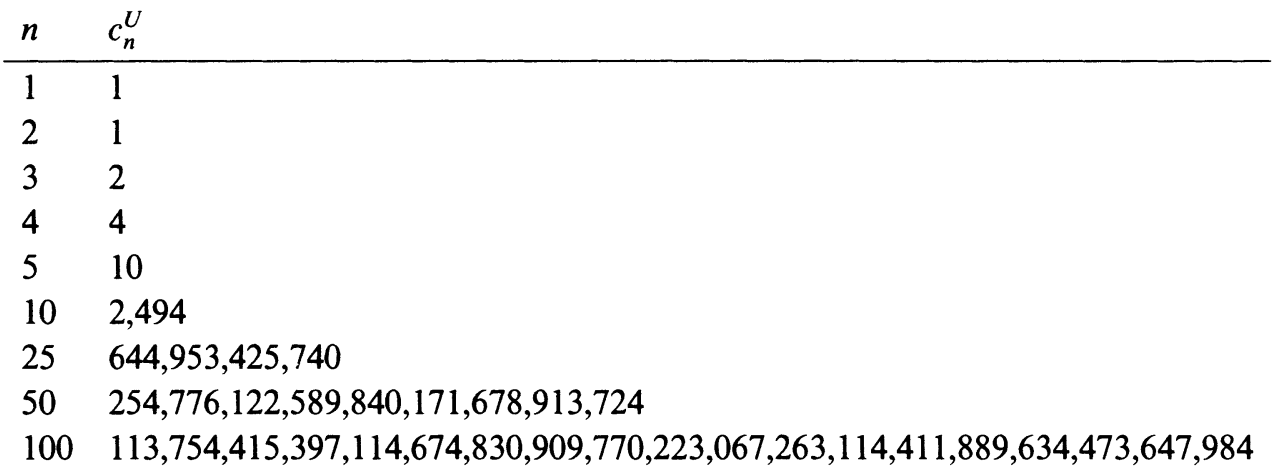

TABLE XIII. Connected unit interval graphs

\begin{tabular}{ll}
$n$ & $p_{n}$ \\
\hline 1 & 1 \\
2 & 0 \\
3 & 1 \\
4 & 1 \\
5 & 3 \\
6 & 4 \\
7 & 11 \\
8 & 21 \\
9 & 55 \\
10 & 124 \\
15 & 15,465 \\
25 & $417,080,105$ \\
50 & $124,476,301,340,558,830,800$ \\
100 & $31,535,643,362,749,645,025,947,988,702,097,854,136,261,741$
\end{tabular}

TABLE XIV. Reduced unit interval graphs 


\begin{tabular}{ll}
$n$ & $j_{n}^{U}$ \\
\hline 1 & 1 \\
2 & 0 \\
3 & 0 \\
5 & 0 \\
6 & 2 \\
7 & 4 \\
8 & 15 \\
9 & 36 \\
10 & 108 \\
11 & 276 \\
12 & 771 \\
13 & 2,044 \\
14 & 5,622 \\
15 & 15,204 \\
25 & $427,946,916$ \\
50 & $129,287,193,751,216,858,640$ \\
75 & $59,768,048,531,223,176,355,913,123,240,820$ \\
100 & $32,928,483,201,293,858,575,745,811,983,737,621,147,644,486$ \\
& \\
& $\quad$ TABLE XV. Identity unit interval graphs \\
$n$ & $k_{n}^{U}$ \\
\hline 1 & 1 \\
10 & 108 \\
25 & $417,824,963$ \\
100 & $124,476,301,313,444,580,840$ \\
& $57,338,574,093,219,742,367,150,464,024,636$ \\
& \\
& $31,585,643,362,749,645,025,931,598,374,911,325,644,799,720$ \\
& \\
&
\end{tabular}

TABLE XVI. Connected identity unit interval graphs 


\section{REFERENCES}

1. E. A. Bender, Asymptotic methods in enumeration, SIAM Rev. 16 (1974), 485-515.

2. J. C. Bohland and C. G. Lekkerkerker, Representations of a finite graph by a set of intervals on the real line, Fund. Math. 51 (1962), 45-64.

3. Joel Cohen, The probability of a unit interval graph (to appear).

4. Joel Cohen, János Komlós and Thomas Mueller, The probability of an interval graph and why it matters, Proc. Sympos. Pure Math., vol. 34, Amer. Math. Soc., Providence, R. I., 1979.

5. P. C. Gilmore and A. J. Hoffman, A characterization of comparability graphs and of interval graphs, Canad. J. Math. 16 (1964), 539, 548.

6. Martin Golumbic, Algorithmic graph theory and perfect graphs, Academic Press, New York, 1980.

7. J. R. Griggs, Extremal values of the interval number of a graph. II (to appear).

8. J. R. Griggs and D. B. West, Extremal values of the interval number of a graph. I, J. Combinatorial Theory Ser. B. (to appear).

9. Frank Harary, Graph theory, Addison-Wesley, Reading, Mass., 1972.

10. F. Harary and J. A. Kabell, Interval graphs and generalizations. I. A new look at multiple interval graphs and interval numbers (to appear).

11. F. Harary and E. Palmer, Graphical enumeration, Academic Press, New York, 1973.

12. F. Harary and W. T. Trotter, Jr., On double and multiple interval graphs, J. Graph Theory (to appear).

13. V. Klee, What are the intersection graphs of arcs in a circle? Amer. Math. Monthly 76 (1969), 810-813.

14. G. Pólya, Kombinatorische Anzahlbestimmungen fur Gruppen, Graphen und chemische Verbindungen, Acta Math. 68 (1937), 145-254.

15. F. Roberts, Discrete mathematical models, Prentice-Hall, Englewood Cliffs, N. J., 1976.

16. __ Graph theory with applications to problems of society, SIAM (1978).

17. Indifference graphs, Proof Techniques in Graph Theory, (F. Harary, ed.), Academic Press, New York, 1969, pp. 139-146.

Department of Mathematics, California Institute of Technology, Pasadena, California 91125

Current address: Department of Mathematics, Massachusetts Institute of Technology, Cambridge, Massachusetts 02139 